\title{
Lizenzmodelle rechtswissenschaftlicher Internetzeitschriften
}

\section{Zur vernachlässigten normativen Dimension des „Open Access“}

Hanjo Hamann*

A. Rechtswissenschaftliche Online-Periodika - eine Bestandsaufnahme ....... 86

I. Spezialisierte Informationsintermediäre ....

86

II. Auswertungen anderer Datenbanken 86

III. Liste der 47 Internetzeitschriften .... 90

B. Institutionelle Charakteristika - eine

Durchmusterung .................. 92

I. Herkunft und Gründungsjahre ...... 93

II. Gründungsakteure .................. 94

III. Themenschwerpunkte .............. 95

IV. Erscheinungsrhythmen und Beitragssprachen .................... 96

V. Begutachtungsverfahren ............ 97

C. "Open“ Access - eine urheberrechtliche Klärung
I. Lizenzmodelle: „Offenheit" und „Freiheit" als originär rechtliche Kategorien ...................... 99

II. Statt Open Access: Traditionelle Rechteverteilung ................... 103

III. „Open Access“ mit unklarer Rechteverteilung ...................... 106

IV. „Open Access“ mit rechtlich restriktiver Lizenzierung ................. 107

V. Open Access mit rechtlich freier Lizenzierung ....................... 109

D. Erfahrungsberichte und Best Practices ................................. 110

E. Zusammenfassung und Fazit .......... 110

Wie andere Wissenschaftsdisziplinen, so verfügt auch die Rechtswissenschaft seit gut zwei Jahrzehnten über Online-Periodika (Internetzeitschriften und wissenschaftliche Blogs). Diese Medien wurden bislang kaum wissenschaftlich untersucht. Lange war schon unklar, welche rechtswissenschaftlichen Zeitschriften überhaupt primär und offen im Internet erscheinen (dazu gleich A.). Auf Grundlage einer über drei Jahre hinweg durchgeführten Datenerhebung untersucht der vorliegende Beitrag deshalb zunächst die institutionellen Charakteristika der bisher etablierten rechtswissenschaftlichen Internetzeitschriften (B.). Anhand dieser Anwendungsfälle widmet er sich einer allgemeineren Rechtsfrage, die ausgerechnet juristische Zeitschriftenherausgeber noch erstaunlich selten umtreibt: Welche normativen Anforderungen stellt „Open Access“ eigentlich an Internetzeitschriften? (C.) Der Beitrag leitet anschließend über zu illustrativen Erfahrungsberichten von Herausgebern rechtswissenschaftlicher Online-Periodika im deutschen Sprachraum (D.), die das Feld näher aufklären sollen und aktuelle Praxisstandards („Best Practices“) entwickeln helfen, um Leser zur kritischen Rezeption und potentielle Herausgeber zur Nachabmung anzuleiten.

* Dr. Dr. Hanjo Hamann ist Senior Research Fellow am Max-Planck-Institut zur Erforschung von Gemeinschaftsgütern (Bonn) sowie Mitherausgeber einer Open-Access-Zeitschrift (www.LanguageAndL aw.eu) und eines wissenschaftlichen Mehrautorenblogs (www.RechtsEmpirie.de). Er dankt Thomas Holzhausen, Daniel Hürlimann, Alexander Peukert, Johannes Rux, Jan Sramek, Julia Wildgans sowie einem anonymen Gutachter für wertvolle Hinweise zum Manuskript. 


\section{A. Rechtswissenschaftliche Online-Periodika - eine Bestandsaufnahme}

Online-Periodika der Rechtswissenschaft im deutschen Sprachraum wurden noch bis vor drei Jahren nirgends systematisch erfasst und waren mitunter selbst mithilfe spezialisierter Informationsintermediäre nur vereinzelt auffindbar (I.). Zum 1. August 2019 wurden die verstreuten und mitunter nur für Eingeweihte auffindbaren Daten erstmals zusammengeführt mit Sonderauswertungen allgemeinerer Datenbanken (II.). Dies ergab eine Liste von derzeit 47 rechtswissenschaftlichen Internetzeitschriften im deutschen Sprachraum (III.), wozu hier neben rein deutschsprachigen Ländern (Deutschland, Österreich, Liechtenstein) auch die Schweiz als eines der (mindestens acht) Länder mit Deutsch als weiterer Amts- oder Verwaltungs-, Regional- oder Nationalsprache gezählt wird.

\section{Spezialisierte Informationsintermediäre}

Online-(insbesondere Open-Access-)Periodika werden in verschiedenen Datenbanken erfasst, die aber zumindest bis einschließlich 2015 für die Rechtswissenschaft lückenhaft ausfielen:

1. Die 2011 eröffnete und bis 2017 kontinuierlich ergänzte Open Access Journals Search Engine (OAJSE) verzeichnete - schon wegen ihrer Beschränkung auf englischsprachige Zeitschriften - seit letztmaliger Aktualisierung ihrer Kategorie „Law“ im Jahr 2013 nur zwei Internetzeitschriften aus dem deutschen Sprachraum. ${ }^{1}$

2. Ein seit mindestens 2015 verfügbarer, von Hamburger Medienrechtlerinnen verfasster und seither immer wieder aktualisierter Überblicksbeitrag auf der Göttinger Informationsplattform Open-Access.net verzeichnete bis 2015 nur 11 der schon damals mindestens 31 rechtswissenschaftlichen Internetzeitschriften; ${ }^{2}$ seither wurde nur eine Zeitschrift ergänzt (Stand 1.8.2019), obwohl zwischenzeitlich mindestens ein Dutzend neu entstanden sind.

3. Das erst 2014 eröffnete Directory of Open Access Scholarly Resources (ROAD) des internationalen ISSN-Zentrums weist inzwischen 28 juristische „Ressourcen “ aus Deutschland, Österreich, der Schweiz und Liechtenstein nach. ${ }^{3}$ Zieht man davon allerdings Schriftenreihen ${ }^{4}$ sowie diejenigen Periodika ab, die be-

1 GoJIL und JIPITEC (web.archive.org/20181225210144/oajse.com/subjects/law.html); zu den Kürzeln gleich Tab. 2.

2 U. Verch/J. Wiesner, Rechtswissenschaft, Open-Access.net (web.archive.org/20150425135845/open-a ccess.net/informationen-fuer-verschiedene-faecher/rechtswissenschaft).

3 Suche am 14.7.2019 unter road.issn.org/advancedsearch nach Subject: Social Sciences > Law. Jurisprudence und Country: Germany + Austria + Switzerland + Liechtenstein. Dabei fehlen rechtswissenschaftliche Internetzeitschriften mit anderer Disziplinzuordnung, wie bspw. TiRuP unter Public health and hygiene. Accident prevention.

4 Bspw. die „Studien zu Grund- und Menschenrechten“ (ISSN 1435-9154) und „CEDPAL. Forschungsstelle für lateinamerikanisches Straf- und Strafprozessrecht" (ISSN 2512-7098) sowie die Berichtsreihen des FGW-Themenbereichs „Digitalisierung von Arbeit - Industrie 4.0“ (ISSN 2510-4071 und 2510-4101), des MPI für Sozialrecht und Sozialpolitik (“Social law reports”, ISSN 2366-7893) 
reits eingestellt sind, ${ }^{5}$ in Wirklichkeit keinen Bezug zum deutschen Sprachraum haben ${ }^{6}$ oder höchstens marginalen Rechtsbezug erkennen lassen, ${ }^{7}$ verbleiben wiederum nur zehn Zeitschriften ${ }^{8}$ und vier Blogs. ${ }^{9}$

4. Auch das zur Standardreferenz avancierte Directory of Open Access Journals (DOAJ) dokumentiert - wohl nicht zuletzt aufgrund allzu restriktiver Indizierungskriterien ${ }^{10}$ - bis heute nur acht rechtswissenschaftliche Internetzeitschriften aus dem deutschen Sprachraum. ${ }^{11}$

5. Sogar die 2015 eingerichtete kommerzielle Datenbank oaFindr, ${ }^{12}$ die mittlerweile zum Elsevier-Konzern gehört und sich rühmt, „Tausende von Zeitschriften aufzuführen, die üblicherweise in bestehenden Sammlungen und Findesystemen fehlen ", 13 indizierte bis Ende 2018 erst 13 rechtswissenschaftliche Internetzeitschriften aus Deutschland und der Schweiz. ${ }^{14}$

In allen genannten Quellen fehlten zahlreiche einschlägige und gut eingeführte Internetzeitschriften; etliche waren bis 2016 sogar nirgends erfasst. Um diesem Dokumentationsmangel abzuhelfen, führte der Autor des vorliegenden Beitrags zum 1. September 2016 die Daten der fünf genannten Quellen zusammen und ergänzte sie aus eigener Erfahrung, durch gezielte Google-Suchen, persönliche Kontakte zu den bis dahin identifizierten Zeitschriftenherausgebern und kontinuierliche Beob-

und des Deutschen Instituts für Menschenrechte („Jahresberichte“, ISSN 2367-3982, und „ABC der Kinderrechte“, ISSN 2198-5642).

5 Die "European Integration online Papers (EIoP)" (ISSN 1027-5193) sind seit 2015 nicht mehr erschienen. Die „Zeitschrift für Sozialreform“ (ISSN 2366-0295) ist schon seit 2010 nicht (mehr?) im Volltext online verfügbar.

6 Etwa die Zeitschrift "Laws" (ISSN 2075-471X, dazu Fn. 11) sowie "Health \& Justice" (ISSN 2194-7899), von deren 21 Herausgebern nur drei in Europa (Niederlande und England) ansässig sind, und das "Specialty Journal of Politics and Law" (ISSN 2520-3282), das trotz Postanschrift in Graz nur drei Herausgeber in Europa hat (Albanien und Ankara), drei hingegen - einschließlich des geschäftsführenden Herausgebers - in Baku (Azerbaijan).

7 Etwa das "Balkan journal of interdisciplinary research" (ISSN 2411-9725) und die "Salamanca working papers series" (ISSN 2509-5080).

8 anci.ch, ALJ, fhi, GoJIL, HRRS, JIPITEC, JLL, SPWR, VLR und ZIS - dazu gleich Tab. 1 im Haupttext.

9 “Humanitarianism \& Human Rights” (ISSN 2199-0859), „Rechtswirklichkeit“ (ISSN 2366-3022), „Verfassungsblog“ (ISSN 2366-7044), „Völkerrechtsblog“ (ISSN 2510-2567).

10 Vgl. bspw. Hamann, in diesem Heft, S. 156, zur bisher verweigerten Aufnahme des JLL.

11 Suchanfragen auf www.doaj.org ergaben am 14.7.2019 für Deutschland neben dem schon nach seinem Titel nicht einschlägigen "SERIEs: Journal of the Spanish Economic Association“ und der mehrheitlich außerhalb des deutschen Sprachraums herausgegebenen „Intergenerational Justice Review" (www.igjr.org) nur noch Rg, RiA und GoJIL (t1p.de/qzra), für Österreich ALJ und EJELS (t1p.de/liot), für die Schweiz anci.ch, EJIMEL, sui generis sowie „Laws“ (t1p.de/rgxc), die jedoch nur ihren Verlag in Basel hat, 42 ihrer 43 Herausgeber dagegen außerhalb Kontinentaleuropas.

12 Dazu Pressemitteilung v. 28.10.2015, unter web.archive.org/20151108005142/prnewswire.com/new s-releases/1science-to-disrupt-the-scholarly-scientific-and-technical-publishing-industry-300167917. html.

13 So die Unternehmensinformationen auf www.1science.com/about-us-2 und www.1science.com/1fi ndr.

14 Laut 1 findr-Datenbankauszug übermittelt von Éric Archambault per E-Mail v. 5.11.2018: anci.ch, EJIMEL, fhi, GLJ, GoJIL, HRRS, JIPITEC, JurPC, MIR, Rg, sui generis, ZERL und ZIS. 
achtung des Zeitschriftenmarkts seit 2016. Diese systematische (und seither mehrfach aktualisierte) Datenerhebung sollte alle rechtswissenschaftlichen Internetzeitschriften identifizieren, deren Herausgeber und Redaktionsmitarbeiter wenigstens mehrheitlich im deutschen Sprachraum (DE, AU, LI, CH) tätig sind, unabhängig von der Publikationssprache des Periodikums selbst. Als „Internetzeitschrift“ wurden dabei nur diejenigen verstanden, die erstens ihre Inhalte primär im Internet (also zeitgleich mit einer etwaigen Printfassung) verbreiten - statt lediglich sekundär, d.h. zeitversetzt gegenüber der primären Druckfassung ${ }^{15}$ - und zweitens im Volltext offen zugänglich sind, also ohne besondere Zugangsdaten erreichbar. ${ }^{16}$

\section{Auswertungen anderer Datenbanken}

Zur Abrundung der Datenerfassung wurden zum 1. August 2019 drei weitere Datenquellen abgefragt, die zwar nicht gezielt Online-Periodika (oder auch nur ausschließlich Zeitschriften)erfassen, aber durch gezielte Sonderauswertungen Aufschluss versprachen:

1. Die wissenschaftliche Metasuchmaschine Bielefeld Academic Search Engine (BASE-search.net). Sie verzeichnete zum 1. August 2019 gut 150 Mio. Datensätze für alle denkbaren Arten wissenschaftlicher Publikationen, davon 241.335 in der Rechtswissenschaft. Von diesen waren 64 mit der „Dokumentart: Zeitschrift/Zeitung “ markiert. ${ }^{17}$ Die Liste dieser Suchtreffer umfasste allerdings keine einzige der bis dahin identifizierten juristischen Internetzeitschriften, und auch keine für eine Ergänzung geeigneten Titel. ${ }^{18}$

2. Das Onlineportal des internationalen ISSN-Zentrums. Es enthielt zum 1. August 2019 rund 2,7 Millionen Standardseriennummern für Periodika (continuing resources), davon gut 122.000 aus dem deutschen Sprachraum. ${ }^{19}$ Da das Portal weder gezielt Zeitschriften auszuwählen noch nach primärer Erscheinungsweise oder Fachdisziplin zu selektieren erlaubt, wurden diejenigen Periodika des deutschen Sprachraums abgefragt, die zumindest auch im Internet er-

15 Die Länge des Zeitversatzes blieb außer Betracht - ob fünf Jahre wie bei der „Kritischen Justiz“ (KJ), ein Jahr wie beim „Newsletter Menschenrechte“ (NLMR) und den Zeitschriften „für Chinesisches Recht“ (ZChinR) und „für ausländisches öffentliches Recht und Völkerrecht“ (ZaöRV), 6 Monate wie bei der „Zeitschrift für Landesverfassungsrecht und Landesverwaltungsrecht“ (ZLVR) oder lediglich 2 Monate wie bei der „Hanover Law Review“ (HanLR).

16 Folglich bleiben Periodika außer Betracht, die zwar rein digital, aber nur innerhalb zugangsbeschränkter Datenbanken erscheinen, wie insb. die (kostenpflichtigen) „Neue Juristische Online-Zeitschrift“ (NJOZ) oder ,juris PraxisReporte“ (jurisPR), aber auch die „bis auf Widerruf kostenlosen“, aber gleichwohl abonnementgebundenen „Fremden- und Asylrechtlichen Blätter“ (FABL) unter www.fabl.at (2009-2017).

17 Suchanfrage archiviert unter t1p.de/lwnu.

18 Das Suchergebnis bestand neben etlichen falsch klassifizierten Aufsätzen vor allem aus deutschen Amtsblättern, Newslettern, US-amerikanischen Law Reviews sowie deutschen Rechtszeitschriften, die nicht primär im Internet erscheinen (insb. dms, MRM, NZBau, studere).

19 Erreichbar unter portal.issn.org, Daten von der Startseite (links Mitte: „World“) bzw. erweiterte Suchanfrage. 
scheinen und im Titel einen der Wortbestandteile „recht“ (361), „juris“ (107), „law“ (92) oder „legal“ (11) enthalten. ${ }^{20}$ Dadurch wurden 32 Titel identifiziert, die zu einer rechtswissenschaftlichen Fachzeitschrift gepasst hätten und trotz ausschließlicher Internetpublikation nicht schon von den unter I. genannten Informationsintermediären erfasst worden waren. Die manuelle Durchsicht der 32 zugehörigen Internetauftritte führte zur Ergänzung (nur, aber immerhin) zweier Internetzeitschriften, die zuvor auf keinem anderen Erfassungsweg hatten identifiziert werden können. ${ }^{21}$ Gleichwohl dürften dabei immer noch Internetzeitschriften verpasst worden sein, die ihre Disziplinzugehörigkeit nicht im Titel erkennen lassen, sowie all jene, die auch gedruckt erscheinen. Zu umfangreich wäre sonst die zu sichtende Datenmenge geworden.

3. Die Elektronische Zeitschriftenbibliothek (EZB) der Universität Regensburg. Sie erschloss zum 1. August 2019 gut 116.000 Periodika aus 41 Disziplinen, davon überdurchschnittlich viele (5.617) aus der Rechtswissenschaft. Von diesen wiederum waren 3.745 als im Volltext frei zugänglich gekennzeichnet. ${ }^{22} \mathrm{Da}$ die öffentliche Suchoberfläche keine Beschränkung auf Fachzeitschriften oder Herkunftsländer zulässt, wurde die Liste der 3.745 frei zugänglichen Rechtsperiodika vom Betreiber angefordert ${ }^{23}$ und computergestützt reduziert: Von den 3.745 Periodika wurden zunächst gut einhundert ausgeschlossen, die nur zeitverzögert (mit Embargofrist) zugänglich sind, also keine echten Internetzeitschriften im hier (oben bei Fn. 15) entwickelten Sinn sein können. Von den verbleibenden nutzten 1.249 eine Internetadresse (URL) mit einer zum deutschen Sprachraum gehörenden Domainendung (TLDs .de, .au, .li, .ch). ${ }^{24}$ Etwa jede zweite (643) erschien erstmals nach 1990, kann also plausiblerweise eine Internetzeitschrift sein. Davon bestehen 534 noch heute. ${ }^{25}$ Für 110 dieser Periodika $(20,6 \%)$ wies die EZB eine Standardseriennummer (ISSN) aus. Deren Titeldaten wurden manuell durchgesehen und ggf. auch der hinterlegte Internetauftritt konsultiert. Durch dieses Vorgehen konnte nur noch ein Titel ergänzt werden; ${ }^{26}$ umgekehrt waren fast drei Viertel $(74 \%)$ der zuvor identifizierten Internetzeit-

20 Beispielsuchen vom 29.7.2019 archiviert unter t1p.de/78s9 für *recht" und t1p.de/a5hh für *juris*.

21 HFSt und ReOS.

22 Erreichbar unter rzblx1.uni-regensburg.de, Daten von der Startseite („Rechtswissenschaft“) bzw. Ampel rechts oben.

23 Herzlich gedankt sei dem EZB-Team, namentlich Claudia Reisinger, für die rasche und unkomplizierte Zurverfügungstellung.

24 Durch diesen Selektionsschritt dürfte etwa jede vierte einschlägige Zeitschrift zu Unrecht ausgeschlossen worden sein: Von den bereits vor der EZB-Recherche bekannten 46 Internetzeitschriften nutzten jedenfalls 12 (26\%) keine zum deutschen Sprachraum gehörende Domain: GLJ, VLR, ZIS, ZJS und ZVR (jeweils.com); GB, GoJIL, JIPITEC und JLL (.eu); AJBALS und EJELS (.org); confront (.news).

25 Seit Beginn der vorliegenden Erhebung (2016) wurden 23 eingestellt. Dabei handelte es sich fast durchweg um Amts- und Gemeindeblätter.

26 Ergänzt wurde WiJ, nicht aufgenommen die ebenfalls EZB-indizierte „Zeitschrift für soziale Strafrechtspflege“ (ISSN 1864-5216), die einen für Fachzeitschriften allzu unsteten Erscheinungsrhyth- 
schriften in der EZB auffindbar; nach Vervollständigung und Berichtigung einzelner Datensätze sogar fast $87 \% .{ }^{27}$ Damit darf die Elektronische Zeitschriftenbibliothek als vollständigste Datengrundlage für Studien zum juristischen Zeitschriftenwesen im deutschen Sprachraum gelten - trotz der bisweilen etwas umständlichen Abfrage und Auswertung, die beachtliches Fehlerpotential nach sich ziehen kann (vgl. Fn. 24).

\section{Liste der 47 Internetzeitschriften}

Aus der skizzierten Datenerhebung entstand ein Überblicksbeitrag über „Open Access als Praxis der Rechtswissenschaft" im kollaborativen Handbuch Open Science bei Wikibooks, ${ }^{28}$ der von jedermann überarbeitet werden kann und durch den $\mathrm{Au}$ tor bis heute laufend aktualisiert und erweitert wird. Die vor knapp drei Jahren erstmals angelegte Liste von 22 Zeitschriften ist mit Stand vom 1. August 2019 auf sieben zeitschriftenähnliche Mehrautorenblogs ${ }^{29}$ sowie folgende 47 Internetzeitschriften angewachsen:

Tab. 1: Bis zum 1.8.2019 dokumentierte rechtswissenschaftliche Internetzeitschriften aus dem deutschen Sprachraum.

\begin{tabular}{llcc}
\hline $\begin{array}{l}\text { (sortiert nach) } \\
\text { Kürzel }\end{array}$ & $\begin{array}{l}\text { Zeitschriftentitel } \\
\text { (J. = Journal, Zs. = Zeitschrift) }\end{array}$ & $\begin{array}{c}\text { online } \\
\text { seit }\end{array}$ & ISSN $^{30}$ \\
\hline AJBALS & $\begin{array}{l}\text { Academic J. of Business, Administration, Law and Social } \\
\text { Sciences }\end{array}$ & 2015 & $2410-8693$ \\
anci.ch & Ancilla Iuris - Lagen des Rechts & 2006 & $1661-8610$ \\
ALJ & Austrian Law J. & 2014 & $2409-6911$ \\
BLJ & Bucerius Law J. & 2007 & $1864-371$ X \\
CL & ContraLegem. [seit 2019:] Recht | Philosophie | Kritik & 2018 & $2624-6902$ \\
Cognitio & & 2018 & $2624-8417$ \\
confront & Studentisches Forum für Recht und Gesellschaft & 2016 & $2509-5595$
\end{tabular}

mus aufweist: Die letzten regulären Hefte (43-51) erschienen 2018, 2015/16, 2013, 2011, 2010, 2009 (2x), 2007 (2x); der Erscheinungszeitpunkt der Hefte 1-42 ließ sich gar nicht ermitteln.

27 Alle außer AJBALS, CL, EJELS, OdW, ReOS und SPWR - also 40/46 Internetzeitschriften.

28 H. Hamann, Rechtswissenschaft, in Wikibooks, Handbuch Open Science, online seit 1.9.2016 unter de.wikibooks.org/wiki/Handbuch_Open_Science/_Rechtswissenschaft.

29 Außer den in Fn. 9 genannten noch das „JuWissBlog“ (juwiss.de), „Umweltrechtsblog“ (umweltrechtsblog.at) und „Rechts|Empirie“ (rechtsempirie.de); eine Zwischenform stellt wohl die 2007 begründete Website publiclaw.at dar, die sich als „e-learning und e-science-Plattform“ beschreibt und mit den „publiclaw.at online publications“ (pop) eine Art Internetzeitschrift umfasst (ISSN 2070-5441), die allerdings in den letzten fünf Jahren nur einen einzigen Beitrag (14.12.2017) publiziert hat.

30 Soweit für eine Zeitschrift verschiedene ISSN aufgefunden wurden (kursive Zellen; p-ISSN für die gedruckte bzw. e-ISSN für die digitale Version), führt die Tabelle die e-ISSN an; die jeweils zugehörige p-ISSN ergibt sich aus der in Fn. 28 zitierten Tabelle und der ISSN-Datenbank unter portal.issn.org.

31 Nicht identisch mit der 2011 in Brasilien gegründeten Internetzeitschrift CognitioJuris.com (ISSN 2236-3009). 


\begin{tabular}{|c|c|c|c|}
\hline $\begin{array}{l}\text { (sortiert nach) } \\
\text { Kürzel }\end{array}$ & $\begin{array}{l}\text { Zeitschriftentitel } \\
\text { (J. = Journal, Zs. = Zeitschrift })\end{array}$ & $\begin{array}{l}\text { online } \\
\text { seit }\end{array}$ & ISSN \\
\hline$\overline{\text { DGStZ }}$ & Deutsch-Georgische Strafrechtszs. & 2016 & $2566-5758$ \\
\hline EJELS & European J. of Economics, Law and Social Sciences & 2017 & $2510-0429$ \\
\hline EJIMEL & Electronic J. of Islamic and Middle Eastern Law & 2012 & $1664-5707$ \\
\hline FoR & Forum Recht (gegr. „Anfang der 80er Jahre“) ${ }^{32}$ & 2007 & 0930-6420 \\
\hline fhi & $\begin{array}{l}\text { forum historiae iuris - Internetzs. für Rechtsgeschichte in Eu- } \\
\text { ropa }\end{array}$ & 1996 & $1860-5605$ \\
\hline FreiLaw & Freiburg Law Students J. & 2006 & $1865-0015$ \\
\hline GB & $\begin{array}{l}\text { Der Grüne Bote - Zs. für Lauterkeitsrecht und Geistiges Eigen- } \\
\text { tum }\end{array}$ & 2004 & $1868-114 X$ \\
\hline GJLE & German J. of Legal Education (pausiert 2018/19) & 2014 & $2509-8675$ \\
\hline GLJ & German Law J. & 2000 & 2071-8322 \\
\hline GoJIL & Goettingen J. of International Law & 2009 & $1868-1581$ \\
\hline HFR & Humboldt Forum Recht & 1996 & $1862-8109$ \\
\hline HFSt & Heidelberger Beiträge zum Finanz- und Steuerrecht & 2015 & 2366-0910 \\
\hline HRN & Hamburger Rechtsnotizen (gegr. 2011) 34 & 2017 & $2191-6543$ \\
\hline HRRS & $\begin{array}{l}\text { Onlinezs. für Höchstrichterliche Rechtsprechung zum Straf- } \\
\text { recht }\end{array}$ & 2000 & $1865-6277$ \\
\hline JIPITEC & $\begin{array}{l}\text { J. of Intellectual Property, Information Technology and Elec- } \\
\text { tronic Commerce Law }\end{array}$ & 2010 & $2190-3387$ \\
\hline JLL & International J. of Language \& Law & 2012 & $2194-7414$ \\
\hline JSE & Jura Studium \& Examen & 2011 & 2195-044X \\
\hline JurPC & $\begin{array}{l}\text { Internet-Zs. für Rechtsinformatik und Informationsrecht (gegr. } \\
1985)^{35}\end{array}$ & 1997 & $1615-5335$ \\
\hline KriPoZ & Kriminalpolitische Zs. & 2016 & $2509-6826$ \\
\hline LeGes & Gesetzgebung \& Evaluation (gegr. 1990) ${ }^{36}$ & 2002 & $2624-5477$ \\
\hline MIR & Medien Internet und Recht & 2005 & $1861-9754$ \\
\hline OdW & Ordnung der Wissenschaft & 2014 & 2197-9197 \\
\hline
\end{tabular}

32 So FoR-Redaktion, „Über“ (web.archive.org/20120718132014/forum-recht-online.de/wp/?page_id= 111); ab 1996 wurden erste Hefte online zur Verfügung gestellt: www.forum-recht-online.de/wp/?ca $\mathrm{t}=43$.

33 Nach dem 4. Jg. 2017 ist die nächste Ausgabe erst für 2020 geplant (Chefredakteur, E-Mail v. 24.7.2019).

34 Frühere Jahrgänge (2011-2016) werden 2019 online archiviert (HRN-Redaktion, E-Mail v. 1.8.2019).

35 Die Zeitschrift wurde 1985 als „Informatik und Recht“ (IUR) gegründet (web.archive.org/2019072 5022033/jurpc.de/jurpc/show?id=iur_1986_0000_0001_0001_0002), firmiert seit 1989 als JurPC und erscheint seit 1997 ausschließlich online (in einzeln nummerierten Web-Dokumenten statt durchgehend paginierten Jahrgängen). Alle früheren Jahrgänge stehen ebenfalls online zur Verfügung (web.archive.org/20190725022529/jurpc.de/jurpc/search?view=archiv).

36 LeGes erscheint seit 2018 ausschließlich online - „im Einklang mit der Open-Access-Strategie der Schweizerischen Akademie der Geistes- und Sozialwissenschaften (SAGW)“ (M. Nussbaumer, In eigener Sache, LeGes 2017, S. 398 und 429) -, war aber zuvor schon als Druck- und Internetzeitschrift unter www.leges.ch verfügbar. Den Beginn der Internetverfügbarkeit konnte der Schriftleiter nicht mehr rekonstruieren, aus dem Druckbild ergibt sich aber das Jahr 2002, wobei die Beiträge seit 1990 sogleich retrodigitalisiert wurden (M. Nussbaumer, E-Mail v. 14.7.2019). 


\begin{tabular}{llcc}
\hline (sortiert nach) & Zeitschriftentitel & online & ISSN \\
Kürzel & (J. = Journal, Zs. = Zeitschrift) & \\
\hline Quid? ${ }^{37}$ & Fribourg Law Review & 2014 & $2297-1106$ \\
ReOS & Recht der Osteuropäischen Staaten & 2014 & $2199-6245$ \\
Rg & Rechtsgeschichte - Legal History & 2002 & $2195-9617$ \\
RiA & Recht in Afrika/Law in Africa/Droit en Afrique (gegr. 1998) $)^{38}$ & 2014 & $2363-6270$ \\
RUB RR & Rechtsprechungsreport der Ruhr-Universität Bochum & 2009 & $2364-5539$ \\
Spektrum & Spektrum der Rechtswissenschaft (eingestellt 2018) $)^{39}$ & 2011 & $2221-9862$ \\
SPWR & Spektrum des Wirtschaftsrechts & 2018 & $2663-9823$ \\
StudZR-Wis- & Studentische Zs. für Rechtswissenschaft Heidelberg: Wissen- & 2014 & $2629-7132$ \\
sOn & schaft Online & & \\
sui generis & sui-generis.ch - Die juristische Open-Access-Zs. & 2014 & $2297-105 X$ \\
TiRuP & Tierschutz in Recht und Praxis & 2017 & $2523-6156$ \\
VLR & University of Vienna Law Review & 2017 & $2521-3962$ \\
WiJ & Journal der Wirtschaftsstrafrechtlichen Vereinigung & 2012 & $2193-9950$ \\
ZERL & Zs. für Europäische Rechtslinguistik & 2010 & $2191-8074$ \\
ZfL & Zs. für Lebensrecht (gegr. 1992) & 2003 & $0944-4521$ \\
ZIS & Zs. für Internationale Strafrechtsdogmatik & 2006 & $1863-6470$ \\
ZJS & Zs. für das Juristische Studium & 2008 & $1865-6331$ \\
ZR\&I & Zs. für Recht \& Islam & 2015 & $2566-7858$ \\
ZVR & Zs. für Verwaltungsrecht Online & 2012 & $2194-1483$ \\
\hline & & & \\
\hline & & &
\end{tabular}

\section{B. Institutionelle Charakteristika - eine Durchmusterung}

Die in Tabelle 1 aufgeführten Internetzeitschriften sind zwar eine Minderheit (schätzungsweise $5 \%)^{40}$ der rechtswissenschaftlichen Fachzeitschriften. Sie weisen aber dennoch eine beachtliche Variationsbreite hinsichtlich ihrer Herkunft und Gründungsjahre (I.), Gründungsakteure (II.), Themenschwerpunkte (III.), Erscheinungsrhythmus und Beitragssprachen (IV.) sowie Begutachtungsverfahren (V.) auf. Daher erschien ihre systematische Durchmusterung durchaus lohnenswert.

37 Nicht identisch mit der 2005-2016 in Mexiko herausgegebenen Internetzeitschrift Quid Iuris (ISSN 1870-5707, unter revistas-colaboracion.juridicas.unam.mx/index.php/quid-iuris).

38 Die unter gleicher Schriftleitung und beim gleichen Verlag herausgegebene „KAS African Law Study Library" wurde nicht berücksichtigt, weil acht ihrer zehn Herausgeber auf dem afrikanischen Kontinent tätig sind; die Zeitschrift „veröffentlicht vorwiegend Artikel junger afrikanischer Wissenschaftler [...] aus Seminaren und Konferenzen, die das Rechtsstaatsprogramm Afrika Südlich der Sahara der Konrad-Adenauer-Stiftung organisiert." (web.archive.org/20190724040126/african-law.nomos. de/autorenhinweise).

39 Dazu web.archive.org/20190724055745/spektrum-der-rechtswissenschaft.at, teilweise fortgesetzt als SPWR (nächste Tabellenzeile).

40 Eine Schätzung ging 2014 von bis zu 1.400 juristischen Fachzeitschriften aus (H. Hamann, Die Fußnote, das unbekannte Wesen, RW 2014, S. 501 [516]); die Wiederholung der damaligen Auswertung mit aktuellen Daten und ohne die als „eingestellt“ gekennzeichneten Periodika ergibt eine genauere Schätzung von 1.130 aktiven Periodika (inkl. Amtsblätter, periodische Sammlungen, etc.). In einer daraus gezogenen $3 \%$-Zufallsstichprobe (34 Titel) wurden 22 Titel als Fachzeitschriften identifiziert; was hochgerechnet auf eine Gesamtzahl von 864 rechtswissenschaftlichen Fachzeitschriften schließen lässt. 47 Internetzeitschriften entsprechen dann 5,4 \%. 


\section{Herkunft und Gründungsjahre}

Von den 47 Zeitschriften haben 33 ihren Redaktionsschwerpunkt in Deutschland (70,2 \%), sieben in der Schweiz (anci.ch, CL, Cognitio, EJIMEL, LeGes, Quid?, sui generis), sechs in Österreich (AJBALS, ALJ, EJELS, Spektrum, TiRuP, VLR), eine in Liechtenstein (SPWR).

Schon seit 2011 verfügte Österreich über eine rechtswissenschaftliche Internetzeitschrift (Spektrum), ${ }^{41}$ die jedoch zum Jahreswechsel 2018/19 wieder eingestellt wurde und deshalb im Weiteren (ab II.) außer Betracht bleibt. Von den noch bestehenden österreichischen, liechtensteinischen und schweizerischen Zeitschriften waren 2011 nur zwei schweizerische aktiv (LeGes und anci.ch). Eine weitere entstand 2012 (EJIMEL), die übrigen zehn in den vergangenen fünf Jahren. In Deutschland erfolgten die letzten Neugründungen ${ }^{42}$ im Jahr 2016 (confront, DGStZ und KriPoZ). ${ }^{43}$ Die ersten hingegen datieren schon auf zwanzig Jahre zuvor, als 1996 gleich zwei Internetzeitschriften an der Humboldt-Universität zu Berlin ins Leben gerufen wurden (HFR ${ }^{44}$ und fhi). ${ }^{45}$ Mit JurPC konvertierte kurz darauf die erste vom Printmedium ins digitale Format. ${ }^{46}$ Weitere zwei Internetzeitschriften entstanden um die Jahrtausendwende (GLJ und HRRS), die meisten späteren Gründungen verteilen sich recht gleichmäßig über den Zeitraum 2005-2014. Dies ergab insgesamt 19 neue Internetzeitschriften über zehn Jahre hinweg. ${ }^{47}$

Insgesamt ergibt sich damit auf den ersten Blick eine recht kontinuierliche Gründungskonjunktur über die letzten 22 Jahre: Durchschnittlich wurden im deutschen Sprachraum jedes Jahr etwas mehr als zwei rechtswissenschaftliche Internetzeitschriften gegründet. Seit 1996 kam es nur sieben Mal vor, dass nicht $2 \pm 1$ Zeitschriften erstmals primär im Internet erschienen; positiv stechen nur die Jahre

41 Unberücksichtigt bleibt der 2007 gegründete NewJurist.com, der sich als „International Law Magazine" beschreibt und nicht primär Wissenschaftler anzusprechen scheint.

42 Als „(Neu-)Gründung“ in diesem Sinne wird auch die bloße Umstellung einer vormals reinen Druckzeitschrift auf eine (auch) im Internet erscheinende Zeitschrift verstanden.

43 Noch später (2017) gaben die 2011 gegründeten HRN ihre erste Internetausgabe heraus.

44 HFR datiert sein Bestehen auf „seit 1995“ (web.archive.org/20190308173040/humboldt-forum-rec ht.de), verfügt aber erst seit 1996 über publizierte Beiträge.

$45 \mathrm{Zu}$ den „Ausgangsüberlegungen der Gründer 1996“ (Internationalität des Diskurses, Kostendruck, Erstarrungstendenzen im Printmedium) ausf. H.-P. Haferkamp u.a., Das 'Forum Historiae Iuris' eine elektronische Zeitschrift zur Rechtsgeschichte, zeitenblicke 2 (2003), Nr. 2 (web.archive.org/20 031210210937/zeitenblicke.historicum.net/2003/02/haferkamp.htm).

46 Dazu schon Fn. 35 sowie Redaktion JurPC, Ein viertel Jahrhundert Rechtsinformatik - 26 Jahre JurPC, JurPC 146/2012 (doi.org/10.7328/jurpcb2012279142).

47 Nicht berücksichtigt wurden Gründungen dieses Zeitraums, die 2016 bereits wieder eingestellt waren. Dazu gehören etwa die „Hanse Law Review“ (HLR, 2005-2014 unter hanselawreview.eu), das 2006 zu Ausbildungszwecken initiierte „Journal of Academic Legal Studies“ (JOALS), für das nur zwei Ausgaben nachweisbar sind (web.archive.org/20080125113100/joals.org), die „pop“ 20082014 (oben Fn. 29); faktisch eingestellt sind wohl auch der 2007 gegründete Kunstrechtsspiegel (ISSN 1864-5690), dessen aktuellster wissenschaftlicher Beitrag von Mai 2016 datiert; etwa zeitgleich erschien das bisher letzte Heft der ZFWIS, „Zeitschrift für angewandte Fragen zu Wirtschaftsprüfung, Immobilienbewertung und Steuerrecht“ (ISSN 2365-5445). 
2012 und 2017 (mit jeweils vier Gründungen) und 2014 (mit acht) heraus. ${ }^{48} \mathrm{Da}$ zwischen 2012 und 2014 allerdings ein Jahr ohne Gründungen lag, fielen sie im Mehrjahresdurchschnitt gar nicht so ungewöhnlich aus, sondern fügen sich in einen allgemeinen Trend allmählich zunehmender Gründungsaktivität:

Abb. 1: Gründungsjahre aller 47 rechtswissenschaftlichen Internetzeitschriften im deutschen Sprachraum (linke Achse), jeweils mit Anzahl der erstmals primär im Internet veröffentlichten Zeitschriften (untere Achse) aus Deutschland (DE), der Schweiz (CH), Österreich (AU) und Liechtenstein (LI).

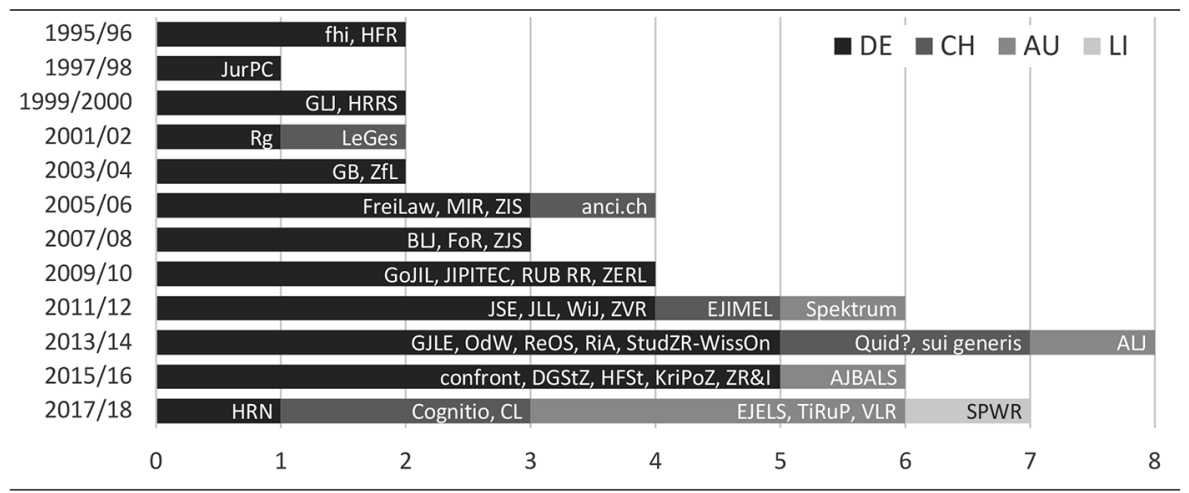

\section{Gründungsakteure}

$\mathrm{Zu}$ den aktivsten Gründern von Internetzeitschriften gehören Nachwuchswissenschaftler, die für 12 der 46 noch bestehenden Zeitschriften verantwortlich zeichnen. Die österreichische VLR und die schweizerische sui generis wurden von Doktoranden gegründet; ein weiteres Viertel der schweizerischen und deutschen Internetzeitschriften von Studierenden. ${ }^{49}$ Die meisten davon werden als Experimentierund Übungsfelder von einer Studierendengeneration an die nächste weitergegeben und sind deshalb stark auf ihre jeweilige Heimatinstitution ausgerichtet. ${ }^{50}$ Mit GoJIL und sui generis gehören aber auch mindestens zwei breiter rezipierte Fachzeitschriften dazu.

Eine zweite relevante Akteursgruppe sind Rechtspraktiker. So wurden die Zeitschriften confront, HRRS, MIR, RiA, WiJ und ZVR maßgeblich von Rechtsanwälten gegründet und nach wie vor betrieben. Auch die Zeitschrift LeGes ist als designiertes „Mitteilungsblatt der Schweizerischen Gesellschaft für Gesetzgebung

48 Negativ dagegen die Jahre 1998, 1999, 2001 und 2013, in denen keine Zeitschrift gegründet wurde.

49 In der Schweiz Cognitio und Quid?, in Deutschland BLJ, FoR, FreiLaw, GoJIL, GJLE, HFR und StudZR-WissOn.

50 FreiLaw publiziert primär Beiträge aus Freiburg, HRN will „das wissenschaftliche Leben an der Fakultät [Hamburg] nach außen repräsentieren“" und „,vor allem studentischen Arbeiten eine Veröffentlichungsplattform [.] bieten“, Quid? spricht ausschließlich Studierende der Universität Fribourg an, VLR stellt nur an der Universität Wien entstandene Forschung vor. 
(SGG) und der Schweizerischen Evaluationsgesellschaft (SEVAL)“ stark in der Praxis verwurzelt, wenngleich sie sich als „wissenschaftliche Zeitschrift“ interdisziplinären Zuschnitts versteht, ${ }^{51}$ und auch durch die Schweizerische Akademie der Geistes- und Sozialwissenschaften (SAGW) gefördert wird. ${ }^{52}$

Die übrigen 27 Internetzeitschriften (20 deutsche, 4 österreichische, 2 schweizerische, 1 liechtensteinische) stammen von Wissenschaftlern: Drei widmen sich Ausbildungszwecken (JSE, RUB RR, ZJS), die übrigen dürfen als wissenschaftliche Fachzeitschriften im engsten Sinn gelten.

\section{Themenschwerpunkte}

Beschränkt man die Auswertung auf die 33 nicht unmittelbar ausbildungsbezogenen Zeitschriften, finden sich zunächst vier ohne Beschränkung auf ein konkretes Rechtsgebiet: „German Law Journal“ (GLJ), „Austrian Law Journal“ (ALJ) und die schweizerischen „sui generis“ und „ContraLegem“ (CL). Hinzu kommen drei Zeitschriften, die sich ebenfalls nicht nach thematischen, sondern nach geographischen Gesichtspunkten definieren: RiA zum Recht Afrikas, ReOS zum Recht Osteuropas sowie GoJIL zum Völkerrecht.

Unter den rechtsgebietsspezifischen Zeitschriften wiederum dominieren das Strafrecht mit sieben Titeln (confront, DGStZ, HRRS, KriPoZ, WiJ, ZfL, ZIS), das Medien- und Urheberrecht mit vier (GB, JIPITEC, JurPC, MIR), gefolgt von Rechtsgeschichte (fhi, Rg) und islamischem Recht (EJIMEL, ZR\&I) mit je zwei Titeln. Hinzu kommen Zeitschriften zu Rechtstheorie (anci.ch), Gesetzgebungslehre (LeGes) und Wissenschaftsrecht (OdW) sowie Finanz- und Steuerrecht (HFSt), Wirtschafts(SPWR), Verwaltungs- (ZVR) und Tierschutzrecht (TiRuP).

Schließlich sind vier Zeitschriften interdisziplinär angelegt, ${ }^{53}$ also keinem Rechtsgebiet verpflichtet, sondern der Kooperation mit Nachbardisziplinen der Rechtswissenschaft. Hierzu gehören die Linguistik (JLL, ZERL) sowie Wirtschafts- und Sozialwissenschaften (AJBALS, EJELS). Allerdings werden die beiden letztgenannten Zeitschriften von der gleichen Einrichtung herausgegeben (IIPCCL, Graz/Tira-

51 So die Selbstbeschreibung, und weiter: „Beiträge aus Rechts- und Politikwissenschaft, Linguistik, Soziologie und Evaluationsforschung sind darin ebenso enthalten wie Berichte über laufende oder abgeschlossene Projekte der Rechtsetzung.“ (web.archive.org/20190311003930/weblaw.ch/compete nce/editions_weblaw/leges.html).

52 Nussbaumer (Fn. 36).

$53 \mathrm{Zu}$ LeGes bereits oben Fn. 51; sie wird hier zur Gesetzgebungslehre als juristischer Kerndisziplin gezählt. 
na), sind als einzige für Autoren kostenpflichtig (APC $)^{54}$ und wecken im Außenauftritt Zweifel an ihrer wissenschaftlichen Seriosität. ${ }^{55}$

\section{Erscheinungsrhythmen und Beitragssprachen}

Wie sich aus der nachstehenden Abbildung ergibt, verzichtet etwa ein Drittel der Zeitschriften (15) auf den unterjährigen Erscheinungsrhythmus und publiziert Artikel entweder einmal jährlich oder kontinuierlich im Jahresverlauf. Ein weiteres Drittel (14) erscheint zwei bis drei Mal im Jahr, das letzte Drittel (15) noch häufiger, wobei etwa die Hälfte davon (7) einmal im Quartal und nur eine Zeitschrift (JurPC) wöchentlich erscheint. Zwei Zeitschriften hingegen lassen gar keinen festen Erscheinungsrhythmus erkennen (HFSt, ZVR). ${ }^{56}$

Genau die Hälfte der Zeitschriften (23) erscheint ausschließlich auf Deutsch, ein gutes Sechstel (8) ausschließlich auf Englisch. Das verbleibende Drittel ist zwei- bis sechssprachig. ${ }^{57}$ Davon lassen nur zwei Zeitschriften kein Englisch zu (DGStZ, LeGes), eine weitere (CL) „alle Sprachen, die wir verstehen“ (derzeit fünf)..$^{58}$ Die beiden liberalsten öffnen sich sogar für „alle größeren Sprachen der Welt“ (Rg) bzw. alle Sprachen der 24 im internationalen Beirat vertretenen Länder (ZIS). Von diesen letztgenannten räumt ZIS allerdings „Englisch, Französisch, Italienisch und Spanisch eine gewisse Präferenz ein “59 und auch eine kursorische Sichtung des Rg-

54 Zum Begriff gleich Fn. 100. Beide Zeitschriften berechnen 200 Euro pro Einreichung zzgl. 80 Euro je Koautor. Auch ReOS verlangt von ukrainischen und russischen Autoren einen „Publikationsbeitrag“ (публикационного вноса, Quelle wie Fn. 61), der aber nur „die Hilfe der Redaktion“ bei „Übersetzung, Korrektur usw.“ abdeckt, soweit der Autor ihrer bedarf (so ReOS-Redaktion, E-Mail v. 5.8.2019).

55 Vgl. Selbstbeschreibungen beider Zeitschriften: "AJBALS is interested in promoting the exchange of idea, and to bring together researchers and academics from all over the countries. [...] Aiming at scientific excellence and quality, this journal will promote the best inputs in scientific- and academic research, on the prospective of local, international, global developments in business, economics, law, social-, but also natural sciences." (web.archive.org/20190308174605/iipccl.org/?page_id=2 388 , Hervorhebungen nur hier); "EJELS aims to promote rapid communication and exchange between the World as well as other Research Scholars, Universities and academic institutions. It mainly reflects the latest advances being made in many disciplines of scientific research in universities and academic institutions." (web.archive.org/20190308174708/iipccl.org/?page_id=4488).

56 ZVR hatte jährlich mindestens vier Ausgaben (2017), bisweilen aber bis zu 18 (2013). HFSt hatte bisher 1-4 Ausgaben pro Jahr, beschreibt sich aber (auch) als „Schriftenreihe“, dürfte also eine Zwischenform darstellen.

57 Neben Mischungen aus Deutsch und Englisch (ALJ, HRN, TiRuP, ZERL, ZR\&I) finden sich zusätzlich auch Französisch (OdW, RiA) und Italienisch (Quid?), zudem noch Spanisch (fhi, CL) und als sechste Sprache Portugiesisch (anci.ch). LeGes nutzt die vier Schweizer Amtssprachen; zu DGStZ, $\mathrm{ReOS}$ und Rg gleich im Haupttext.

58 Redaktion, Zur Kritik des aktuellen Zustandes der Wissenschaft, ContraLegem 2018, S. 4 (8): „d.h. zumindest Texte in Deutsch, Französisch und Englisch. Auch italienische oder spanische Texte sind willkommen, wenn der Autor tatsächlich glaubt, auf diese Weise genügend Leser zu erreichen, was wir im schweizerischen Kontext allerdings bezweifeln. Im Falle von Romantsch brauchen wir eine Übersetzung, ohne die wir nicht verstehen würden, was wir veröffentlichen." (web.archive.org/2019 0310161542/contralegem.ch/2018-1-editorial).

59 ZIS-Redaktion, International Advisory Board (web.archive.org/20190730081925/zis-online.com/in dex.php?sektion=4\&sektionsub=10); vgl. auch Th. Rotsch, in diesem Heft, S. 125 f.: „regelmäßig in 
Archivs förderte nur Texte auf Deutsch, Englisch und romanische Sprachen zutage, während keine Beiträge auf Arabisch, Chinesisch oder Russisch zu finden waren. Nicht-lateinische Schriftsysteme nutzen nur zwei Zeitschriften: DGStZ („stets zweisprachig" auf Deutsch und Georgisch ${ }^{60}$ und ReOS (Ukrainisch und Russisch neben Deutsch und Englisch). ${ }^{61}$

Abb. 2: Erscheinungsrhythmus der 46 rechtswissenschaftlichen Internetzeitschriften (linke Achse: Ausgaben pro Jahr, „var." = variabel), jeweils mit genutzten Beitragssprachen: nur Deutsch (DE), nur Englisch (EN) oder mehrsprachig (MS).

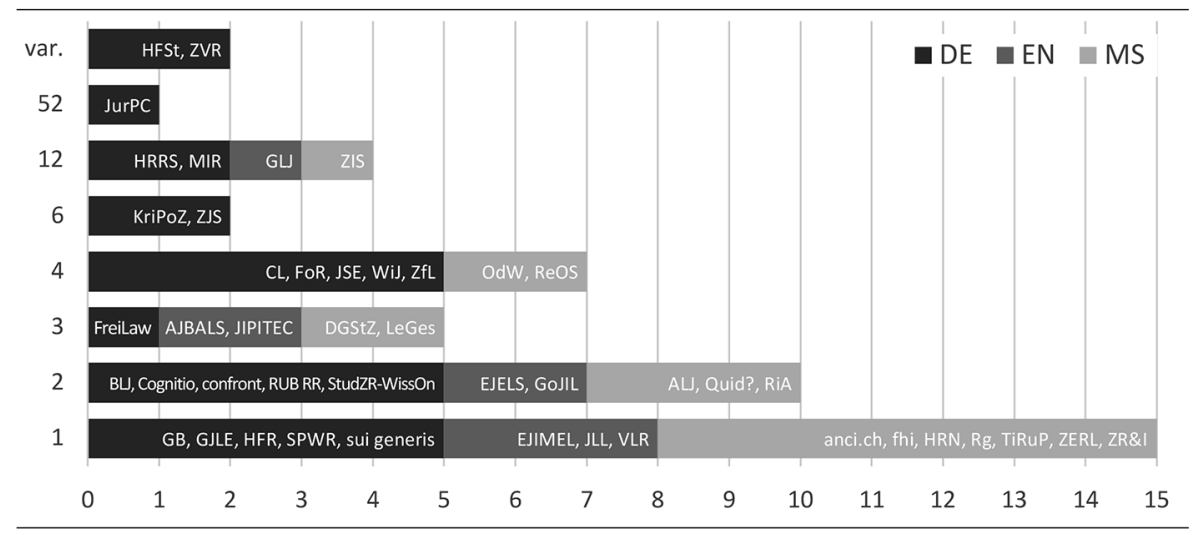

\section{Begutachtungsverfahren}

Für juristische Zeitschriften bemerkenswert oft kommen Begutachtungsverfahren zum Einsatz: 20 rechtswissenschaftliche Internetzeitschriften geben an, eine Kreuzbegutachtung (Peer Review) durchzuführen, ${ }^{62}$ die in den meisten Fällen zusätzlich als doppelt-blind bezeichnet wird. Zwei weitere Zeitschriften (TiRuP, ZIS seit 2010) nutzen die Kreuzbegutachtung zumindest selektiv ${ }^{63}$ und nur eine Zeitschrift (CL) spricht sich grundsätzlich gegen Peer-Review-Verfahren aus. ${ }^{64}$

deutscher, englischer und spanischer Sprache. Daneben wurden Beiträge auf Portugiesisch, Französisch, Italienisch, Griechisch und Rumänisch veröffentlicht".

60 DGStZ-Redaktion, Konzept, verlinkt unter www.dgstz.de/page/proektis-shesakheb (als pdf), S. 2.

61 ReOS-Redaktion, Для Авторов [für Autoren]: „язык статьи украинский, русский, английский или немецкий“ (web.archive.org/20170310142248/reos.uni-goettingen.de/?page_id=118).

62 AJBALS, ALJ, anci.ch, EJELS, EJIMEL, GJLE, GLJ, GoJIL, JIPITEC, JLL, JSE, KriPoZ, Rg, RiA, SPWR, sui generis, VLR und ZERL.

63 So TiRuP-Redaktion, Über die Zeitschrift: „Je nach Themen- bzw Fragestellung entscheiden die Herausgeber/-innen gegebenenfalls über die Durchführung einer spezifischen fachlichen Begutachtung (zB double-blind Peer Review)“ (web.archive.org/20190724090545/eplus.uni-salzburg.at/tirup /wiki/about); ZIS-Redaktion, Hinweise für Autoren: „Sämtliche fremdsprachigen Aufsätze werden im International Advisory Board im peer review-Verfahren anonym evaluiert.“ (web.archive.org/20 100612070311/zis-online.com/?sektion=4\&sektionsub=2\&language=ger).

64 Redaktion, Zustand (Fn. 58), S. 5: „Wir glauben deshalb nicht an Peer-Reviewing, das in unseren Augen nur Standard, nur Normalwissenschaft produziert" usw. 
Die mit fast $50 \%$ ungewöhnlich hohe Verbreitung von Peer Review unter rechtswissenschaftlichen Internetzeitschriften überrascht zunächst angesichts der landläufigen Skepsis gegenüber Begutachtungsverfahren in der Rechtswissenschaft. ${ }^{65}$ Erklären lässt sie sich vielleicht aus (einer Kombination von) dem vergleichsweise geringen Alter von Internetzeitschriften, ihrem Modernitätsanspruch und Innovationsdrang, ihrer weltweiten Sichtbarkeit, die einen Anschluss an internationale Wissenschaftsgepflogenheiten ratsam erscheinen lässt, ${ }^{66}$ oder mit einem gesteigerten Bedürfnis nach Qualitätsauslese, um der vielgescholtenen Beliebigkeit des Online-Publikationswesens entgegenzutreten. Was auch immer der Grund sein mag,bestätigt die vorliegende Durchmusterung jedenfalls den schon fast zehn Jahre zuvor berichteten empirischen Befund, „dass anteilig mehr Open-Access-Zeitschriften eine Qualitätssicherung der Artikel in Form eines Peer-Review-Verfahrens vornehmen als traditionelle Printzeitschriften. “67

Fraglich bleibt freilich, inwieweit die von rechtswissenschaftlichen Internetzeitschriften behauptete „Peer Review“ tatsächlich internationalen Gepflogenheiten entspricht und den als Vorbild herangezogenen Publikationsstandards quantitativer Wissenschaften nicht nur rhetorisch Reverenz erweist. Fast alle hier untersuchten Zeitschriften bleiben vage bei der Beschreibung dessen, was sie als Peer Review verstehen. Nur eine Zeitschrift (JLL) beschreibt detailliert ihr Verfahren und die Kriterien für eine Beurteilung durch externe Gutachter. ${ }^{68}$

Soweit die anderen ihr Verfahren erahnen lassen, fehlt es teils schon an der Einbindung externer Gutachter, ${ }^{69}$ wenn die Begutachtung bspw. durch einen ständigen Beirat (JSE), einen „Gutachterrat“ (ZERL), ein „International Advisory Board“ (ZIS) oder ein (immerhin sehr umfangreiches) ${ }^{70}$ „Peer Review Board“ erfolgt (sui

65 Statt aller P. Kostorz, Review-Verfahren bei der Veröffentlichung juristischer Fachartikel - Was soll das?, KJ 2016, S. 417; Zivilrechtslehrervereinigung, Kriterien für die Beurteilung wissenschaftlicher Leistungen pp., 2015, S. 4 (Rn. 14): „der Umstand, dass der Annahme zur Veröffentlichung ein peer-review vorausgegangen ist“ belege keine „überdurchschnittliche wissenschaftliche Qualität.“ (web.archive.org/20190731213415/zlv-info.de/fileadmin/PDF/Leitlinien/Qualitaetskriterien_fuer_di e_Beurteilung_wissenschaftlicher_Leistungen.pdf); offener jüngst K. Purnhagen / N. Petersen, Evaluation of Academic Legal Publications in Germany, in R. van Gestel / A. Lienhard (Hrsg.), Evaluating Academic Legal Research in Europe, 2019, unter 8.

66 So meint etwa die SPWR-Redaktion, „dass nicht ausgeschlossen werden kann, dass für die akademischen Karrieren von jungen Juristinnen und Juristen in Zukunft die Frage, in welchen Zeitschriften publiziert wurde und wie deren Auswahlverfahren ausgestaltet ist, einen ähnlichen Stellenwert bekommt, wie in anderen Wissenschaften." (web.archive.org/20190724040310/spektrum-des-wirtsch aftsrechts.at/index.php?id=34).

67 A. Oberländer / R. Vock, Open Access. Wissen für Alle!, FoR 2010, S. 15 (16).

68 Vgl. web.archive.org/20190308182924/languageandlaw.eu/jll/about/editorialPolicies\#peerReviewPr ocess.

69 Beispielhaft Purnhagen/Petersen (Fn. 65), unter 4.1.1: "Submissions to the German Law Journal, for example, are usually reviewed anonymously by one member of the editorial board, not external reviewers.”.

7048 Personen laut web.archive.org/20190308182437/sui-generis.ch/peer-review-board. 
generis). Mit dem international etablierten Verständnis einer unabhängig-kriteriengeleiteten Begutachtung sind solche Verfahren ebensowenig vereinbar wie die unklare Kommunikation ihrer Auswahlkriterien. ${ }^{71}$

\section{C. „Open“ Access - eine urheberrechtliche Klärung}

Noch öfter als in der Frage der Peer Review suchen rechtswissenschaftliche Internetzeitschriften den Anschluss an internationale Publikationsstandards durch ein öffentliches Bekenntnis zum Open Access: Genau die Hälfte beschreibt sich - mitunter eher diskret - als „Open Access“..$^{72}$ Dabei übersehen viele Herausgeber, dass „offener" Zugang neben der technischen Abrufbarkeit auch spezifisch normative Anforderungen stellt (I.). Abgesehen also von jener Hälfte der Internetzeitschriften, die von vornherein auf Open Access verzichtet (II.), müssen sich juristische Internetzeitschriften an der von allen deutschen Wissenschaftsorganisationen konsentierten Definition des „Open Access“ messen lassen. ${ }^{73}$ Dabei stellt sich heraus, dass für etliche unklar zu sein scheint, was „Open Access“ eigentlich bedeutet (III.), während andere damit etwas meinen, das mit der anerkannten Definition des „Open Access“ unvereinbar ist (IV.). Nur fünf rechtswissenschaftliche Internetzeitschriften sind deshalb tatsächlich zweifelsfrei Open Access (V.).

\section{Lizenzmodelle: „Offenheit“ und „Freiheit“ als originär rechtliche Kategorien}

Vereinzelte Stimmen im Schrifttum ${ }^{74}$ und nicht wenige rechtswissenschaftliche Internetzeitschriften (bspw. LeGes, SPWR) setzen Open Access mit „kostenlos“ gleich, verbinden damit also vor allem die Vorstellung, dass Artikel „ohne vorherige Anmeldung“ abrufbar seien (ZERL) oder „dass alle Inhalte frei verfügbar sind und keine Gebühren erhoben werden“ (Rg). Nach hier vertretenem Verständnis hingegen ist der technisch unbehinderte kostenfreie Zugang bereits eines der beiden Definitionsmerkmale einer Internetzeitschrift (vgl. oben vor Fn. 16: offen $z u$ gänglich) und fällt auch nach der herrschenden Ansicht im (internationalen) Schrifttum nicht mit dem Begriff „Open Access“ ineins. Vielmehr werden zwei ver-

71 Ähnl. Purnhagen/Petersen (Fn. 65): "there are some further journals that claim to have a peer-review process. However, a closer look reveals that the peer-review procedure often does not conform to international standards. In particular, the 'peers' reviewing the articles are the editors of the journal and the procedure is not blind in either direction.”.

72 Alle (sechs) aus Österreich und Liechtenstein, fünf aus der Schweiz (anci.ch, Cognitio, EJIMEL, LeGes, sui generis), zwölf aus Deutschland (fhi, GLJ, GoJIL, HFSt, JIPITEC, JLL, JurPC, Rg, RiA, WiJ, ZERL, ZR\&I).

73 Diese Feststellung impliziert keine Bewertung der Lizenzierung von Wissenschaftstexten allgemein oder bestimmter Lizenzmodelle insbesondere, sondern soll die Begriffsverwendung am Sprachgebrauch aller großen deutschen Wissenschaftsorganisationen (bei und in Fn. 77) prüfen; ähnl. schon Wildgans, Zitat nach Fn. 123.

74 Etwa F. Knauer, Neue juristische Publikationsformate im Internet, NJOZ 2009, S. 3004; V. Haug, Open Access in Baden-Württemberg, OdW 2019, S. 89; K.G. Saur, Wissenschaftliche Verlage - Versuch einer Zukunftsprognose, in Degkwitz (Hrsg.), Bibliothek der Zukunft - Zukunft der Bibliothek. FS Elmar Mittler, 2016, S. 132 (137). 
schiedene Bedeutungsdimensionen des im Zitat soeben verwendeten Begriffs „frei“ unterschieden: „Freibier“ ist kostenlos erhältlich, „freie Rede“ bedarf dagegen rechtlicher Gewährleistung. ${ }^{75}$

Diese Doppeldeutigkeit des Freiheitsbegriffs führte in der Open-Access-Debatte zur Differenzierung zwischen „gratis Open Access“ (Internetzeitschriften ohne Kostenbarrieren) und „libre Open Access“ (Internetzeitschriften ohne Kosten- und Rechtsbarrieren) ein. ${ }^{76}$ Inzwischen hat sich die „libre“-Variante des Open Access allgemein durchgesetzt. Sie geht zurück auf die Budapest Open Access Initiative aus dem Jahr 2002 und erhielt mit der „Berliner Erklärung über den offenen Zugang zu wissenschaftlichem Wissen" aus dem Folgejahr ihre von allen großen Wissenschaftsorganisationen Deutschlands anerkannte Formulierung. ${ }^{77}$ Nach dieser Erklärung müssen Open-Access-Veröffentlichungen „zwei Voraussetzungen erfüllen“: Sie müssen in geeigneter Weise archiviert sein, ${ }^{78}$ sowie weltweit „allen Nutzern unwiderruflich“ und mit der rechtsverbindlichen Erlaubnis zugänglich, sie „in jedem beliebigen digitalen Medium und für jeden verantwortbaren Zweck zu kopieren, zu nutzen, zu verbreiten, zu übertragen und öffentlich wiederzugeben sowie Bearbeitungen davon zu erstellen und zu verbreiten, sofern die Urheberschaft korrekt angegeben wird. “79

Dieser weite Lizenzumfang - insbesondere die Befugnis zur Bearbeitung - wird im rechtswissenschaftlichen Schrifttum vereinzelt als nicht wissenschaftsadäquat kritisiert. ${ }^{80}$ Dabei steht es Autoren natürlich frei, Zeitschriften mit weniger liberalen Lizenzen zu wählen. Gleichwohl lassen sich schon heute durchaus Bedürfnisse in Forschung und Lehre ausmachen, die liberale Lizenzen erfordern. ${ }^{81}$ Zudem soll innovationsfördernde Lizenzierung gerade auch Zukunftsanwendungen ermöglichen,

75 Differenzierung nach R. Stallman, Free Software, Free Societies, 2002 (online unter web.archive.org /20190724040448/gnu.org/philosophy/fsfs/rms-essays.pdf), S. 7, 43, 59, 65 Fn. 1, 165, zit. in $L$. Lessig, Free Culture, 2004 (online unter web.archive.org/20190706213648/free-culture.cc/freecultu re.pdf), S. xiv; ich danke einem anonymen Gutachter für den Hinweis auf Lessig.

76 P. Suber, Gratis and libre open access, SPARC Open Access Newsletter \#124 v. 2.8.2008 (nrs.harvard.edu/urn-3:HUL.InstRepos:4322580); zugrundegelegt bei A. Peukert, Das Verhältnis zwischen Urheberrecht und Wissenschaft, JIPITEC 2012, S. 142 (149 Rn. 46): „ohne rechtliche und technische Barrieren".

77 Berliner Erklärung v. 22.10.2003 von Wissenschaftsrat, Hochschulrektorenkonferenz, Deutscher Forschungsgemeinschaft, Berlin-Brandenburgischer Akademie der Wissenschaften, Max-Planckund Fraunhofer-Gesellschaft, Leibniz- und Helmholtz-Gemeinschaft u.a., archiviert unter web.archi ve.org/20190308184218/openaccess.mpg.de/68053/Berliner_Erklaerung_dt_Version_07-2006.pdf.

78 Inwieweit die rechtswissenschaftlichen Internetzeitschriften dieses Erfordernis erfüllen, war mit vertretbarem Aufwand nicht überprüfbar.

79 Berliner Erklärung (Fn. 77), S. 2.

80 Bspw. Krujatz, Open Access (Fn. 85), der die Bearbeitungslizenz in der Wissenschaft nicht für interessengerecht hält.

$81 \mathrm{Zu}$ denken ist etwa an Vorlesungsreader jenseits der Schranken von $\mathbb{S} 60 \mathrm{a}$ f. UrhG, Aufsatzsammlungen, die von unabhängigen Drittherausgebern kuratiert werden, oder öffentlich verfügbar gemachte Forschungskorpora, wie sie (bislang nur) für Gerichtsentscheidungen existieren (bspw. www.english-corpora.org/scotus). 
die sich heute vielleicht noch nicht vorstellen lassen. Im Vergleich dazu erscheint das gegen die Bearbeitungslizenz angeführte Plagiats- und Irreführungsrisiko ${ }^{82}$ vernachlässigbar, weil durch hinreichend strenge Auslegung der Pflicht zur Urheberattribution (dazu gleich nach Tab. 2) eine präzise Abgrenzung verschiedener Textstufen oder Autorenbeiträge zu erreichen ist. ${ }^{83}$ Jedenfalls erfordert auch die Diskussion über Bearbeitungslizenzen einen unmissverständlichen Sprachgebrauch; ${ }^{84}$ erst dieser erlaubt überhaupt zu diskutieren, ob „Open Access“ - wie von den deutschen Wissenschaftsorganisationen definiert - für die Rechtswissenschaft interessengerecht erscheint oder nicht. Einer Antwort auf diese Frage kommt jedenfalls nicht näher, wer „Open Access“ von vornherein enger versteht und die für problematisch gehaltenen Aspekte des Themas schon aus dem Begriff „herausdefiniert“.

Mithin bedarf „Open Access“ also nicht nur eines technisch unbehinderten $\mathrm{Zu}$ gangs frei von Kosten (gratis), sondern auch einer spezifisch rechtlichen Freiheitsgewährleistung (liberty), üblicherweise in Gestalt urheberrechtlich abgesicherter Nachnutzungsbefugnisse. Dieses in der deutschen Rechtswissenschaft bislang weithin „unbekannte Wesen des Open Access“ wird noch seltener in eigener Sache, also bezogen auf rechtswissenschaftliche Fachliteratur, thematisiert. ${ }^{85}$ Es genügt danach jedenfalls nicht, „ein Werk einfach als PDF-Datei ins Netz zu stellen. Der Urheber muss vielmehr der Internetöffentlichkeit die von ,Open Access' geforderten Nutzungsrechte ausdrücklich einräumen ", 86 und dies eingedenk der oben zitierten Definition unwiderruflich. Deshalb können Internetzeitschriften, die sich „alle Rechte vorbehalten“ ${ }^{\text {87 }}$ oder die Weiterverbreitung ihrer Inhalte von einer „schrift-

82 Vor Krujatz, Open Access (Fn. 80) schon O. Sosnitza, Google Book Search, Creative Commons und Open Access, RW 2010, S. 225 (239): „Da Open Access [...] Bearbeitungen des Werkes erlaubt, [... könnten] dem Autor solche Inhalte zugerechnet werden, die vom Bearbeiter stammen und mit denen der Autor nicht einverstanden ist.".

83 A.A. M. Fehling, Von der kommerziellen Verlagsproduktion zum Open Access, in Hoffmann-Riem (Hrsg.), Innovationen im Recht, 2016, S. 337 (345): „Beispielsweise kann durch eine schlechte Übersetzung oder durch sachwidrige Kürzungen die Bedeutung eines Textes verfälscht werden. [...] Solche Verfälschungen oder Umwidmungen vermögen bei Weiterverbreitung selbst dann, wenn der Übersetzer/Bearbeiter genannt ist, die Reputation des ursprünglichen Autors (und zugleich die Funktionsfähigkeit des Wissenschaftssystems) zu beschädigen.“.

$84 \mathrm{Zu}$ diesem Anliegen schon oben Fn. 73 und unten bei Fn. 126.

85 Ausnahmen jüngst J. Wildgans, Zuckerbrot oder Peitsche? - Ein Plädoyer für Open Access im juristischen Publikationswesen, ZUM 2019, S. 21 (Zitat dort S. 22) und M. Goller, in diesem Heft, S. 231; früher schon Oberländer/Vock, Wissen für Alle (Fn. 67); allg. M. Bäuerle, Open Access zu hochschulischen Forschungsergebnissen?, in Britz (Hrsg.), Forschung in Freiheit und Risiko, 2012, S. 1; monographisch u.a. S. Krujatz, Open Access, 2012.

86 Bäuerle (vorige Fn.), S. 6.

87 So GLJ, das sich als Open-Access-Pionier seit 20 Jahren sieht, aber noch bis Jahresanfang dieselben Rechte vorbehielt wie Zeitschriften ohne Open-Access-Selbstbild (dazu gleich II.), vgl. Submissions, 20.1.2019: "retains the copyright over its publications" (web.archive.org/20190120151354/german lawjournal.com/submissions). 
lichen Genehmigung“88 abhängig machen, trotz ihres womöglich freien („gratis“) Zugangs kaum als „Open Access“ gelten, weil sie keine freie („libre“) Zugänglichkeit im Sinne unwiderruflich garantierter Nachnutzung herstellen. Am anschaulichsten illustriert dies vielleicht die jüngst gegründete SPWR, die sich zwar ausdrücklich als „Open Access“ versteht, aber dennoch auf jeder einzelnen Zeitschriftenseite den Vermerk „() Jan Sramek Verlag“ platziert ${ }^{89}$ und betont, dass das Abonnement zwar „bis auf weiters kostenlos“ sei, aber in Zukunft „einen Preis haben“ könnte. ${ }^{90} \mathrm{Da}$ der freie Zugang mithin „ganz schnell wieder geschlossen werden“ kann, hängt er etwas überspitzt „von der Gnade des Verlags oder der Plattform ab".91

Daraus folgt also: Was der Öffentlichkeit jederzeit entzogen werden kann, erfüllt schon nicht das Open-Access-Definitionsmerkmal „unwiderruflich“ - ganz zu schweigen von den (ebenfalls unklaren) Nachnutzungsbefugnissen. Damit geht der Blick weg von der Kostenfrage, hin zur Aufteilung von Rechten zwischen Zeitschrift, Autor(inn)en und Nutzer(inne)n. Um solche Rechteverteilungen verständlich und einheitlich zu kommunizieren, entwickeln vor allem zwei gemeinnützige Organisationen vorformulierte Standardlizenzen: ${ }^{92}$ Die Digital Peer Publishing Initiative des Bundeslandes NRW hält einen - ironischerweise urheberrechtlich geschützten - Standardlizenzvertrag (DPPL) mit zwei Zusatzmodulen vor; ${ }^{93}$ die Creative-Commons-Initiative des US-amerikanischen Rechtswissenschaftlers Lawrence Lessig eine Palette von sechs verschiedenen Standardlizenzverträgen (CC-Lizenzen)..$^{94}$

88 So WiJ, Info für Autoren: „Kein Teil des WisteV-Journal darf ohne schriftliche Genehmigung des WisteV-Journal reproduziert oder anderweitig veröffentlicht werden." (web.archive.org/201908011 40154/wi-j.com/info-fuer-autoren).

89 Nach Auskunft des Verlagsgründers „eigentlich nur auf das Layout“ bezogen, um kommerziellen Nachdruck zu verhindern; eine Umstellung auf CC BY-NC ab 2020 wird erwogen (Jan Sramek, EMail v. 29.7.2019).

90 So die SPWR-Redaktion, „Hilfe/FAQ“, zit. in Fn. 66 a.E.; ähnlich zuvor schon die „Spektrum der Rechtswissenschaft“ (2011-2018, dazu Fn. 39): web.archive.org/20150222093504/spektrum-der-re chtswissenschaft.at/index.php?id=34\#c57.

91 So Goller (Fn. 85), S. 231, noch ohne Kenntnis der SPWR.

92 Ausf. R. Mantz, Open Access-Lizenzen und Rechtsübertragung bei Open Access-Werken, in G. Spindler (Hrsg.), Rechtliche Rahmenbedingungen von Open Access-Publikationen, 2006, S. 55-103.

93 HBZ, Die Digital Peer Publishing Lizenzen, web.archive.org/20190310225326/hbz-nrw.de/produkt e/open-access/lizenzen/dppl.

94 Creative Commons, Mehr über die Lizenzen (web.archive.org/20190310225523/creativecommons. org/licenses/?lang=de); dazu ausf. M. Deingruber, Kreativität für Alle. Creative Commons: Mehr Inspiration durch neue Lizenzformen, FoR 2010, S. 13 (web.archive.org/20190424104800/forum-re cht-online.de/wp/?p=545); knapp auch Sosnitza, Creative Commons (Fn. 82), S. 233 f. 
Letztere sind in der Praxis gebräuchlicher und seien deshalb durch folgende Tabelle näher erläutert. ${ }^{95}$ Die Tabelle wird durch zwei wertbildende Befugnisse des Autors aufgespannt: Die Befugnis zur kommerziellen Verwertung seines Originaltextes und die Befugnis zur Verwertung eines abgeleiteten Textderivats. Beide Befugnisse kann der Autor entweder sich selbst vorbehalten oder an seine Leser/Nutzer lizensieren, ggf. auch unter der Bedingung, dass der Lizenznehmer eine gleichlautende Lizenz am eigenen Werk einräumt (reziprok bedingt). Aus der Kreuztabellierung dieser Möglichkeiten ergeben sich sechs Lizenzinhalte:

Tab. 2: Überblick über die sechs Creative-Commons-Lizenzen (CC). „BY“ steht für die Pflicht zur Urheberattribution, „NC“ für Non-Commercial, „ND“ für No Derivatives, „SA“ für Share Alike. ${ }^{96}$

\begin{tabular}{|c|c|c|c|}
\hline \multirow{2}{*}{$\begin{array}{c}\text { Kommerzielle Ver- } \\
\text { wertung? }\end{array}$} & \multicolumn{3}{|c|}{ Verwertung von Textderivaten? } \\
\cline { 2 - 4 } & unbedingt vorbehalten & reziprok bedingt & $\begin{array}{c}\text { unbedingt einge- } \\
\text { räumt }\end{array}$ \\
\hline vorbehalten & BY-NC-ND & BY-NC-SA & BY-NC \\
\hline eingeräumt & BY-ND & BY-SA & BY \\
\hline
\end{tabular}

Alle Lizenzen setzen die korrekte Urheberangabe (Attribution) voraus, was durch das Kürzel „BY“ kenntlich gemacht ist. Verzichtet der Urheber auch darauf, so wird die einfache „BY“-Lizenz (rechte untere Zelle) zur bedingungslosen Lizenz CCO („Zero“), die eine Nachnutzung des Textes annähernd im gleichen Umfang ermöglicht, als wäre er gemeinfrei entstanden.

Gleicht man diese Lizenzstruktur mit der oben (bei Fn. 79) zitierten Open-AccessDefinition ab, so zeigt sich, dass nur die beiden liberalsten Lizenzen „BY-SA“ und „BY“ die eingangs genannten Anforderungen der deutschen Wissenschaftsorganisationen erfüllen, ${ }^{97}$ die übrigen vier Lizenzen folglich auch nicht als „Open Access" bezeichnet werden dürften. Hierauf wird im Weiteren noch einzugehen sein, wenn sogleich die einzelnen Lizenzmodelle aller rechtswissenschaftlichen Internetzeitschriften näher untersucht werden.

\section{Statt Open Access: Traditionelle Rechteverteilung}

Einige Internetzeitschriften verzichten von vornherein auf eine Lizenzierung, weil sie parallel zu ihrer Internetfassung auch gedruckt erscheinen. Herkömmlich ge-

95 Ich danke einem anonymen Gutachter für die Anregung. Alternative Illustration als Flussdiagramm bei J. Muиß-Merholz, Die CC-Lizenzen im Überblick - Welche Lizenz für welche Zwecke?, wb-web, web.archive.org/20190310224055/wb-web.de/material/medien/die-cc-lizenzen-im-uberblick-welchelizenz-fur-welche-zwecke-1.html.

96 Näher U. Herb, Creative-Commons-Lizenzen und Open-Access-Zeitschriften, JurPC 5/2015 (doi.org/10.7328/jurpcb20153011).

97 Schon für Krujatz, Open Access (Fn. 85), S. 135 „korrespondieren lediglich das CC-Lizenzmuster ,Namensnennung' sowie die ,Freie DPPL' vollends mit der hier zugrunde gelegten Definition des Open-Access-Prinzips. “. 
druckte Zeitschriften rechnen sich zu den heute üblichen Abonnementpreisen nur dann, wenn neben ihren Nutzern ${ }^{98}$ auch die Autoren einen Teil der Herstellungsund Vertriebskosten übernehmen. ${ }^{99}$ Da Autoren bisher nicht zu einer Kostenbeteiligung in Geld bereit sind, ${ }^{100}$ beteiligen sie sich traditionell durch weitgehenden (mitunter vollständigen) Verzicht auf Vergütungen oder Tantiemen für die Verwertungsrechte. ${ }^{101}$ Diese werden dem Verlag exklusiv und meist medienübergreifend, weltweit und für die gesamte Dauer des Urheberrechts eingeräumt. Diese traditionelle Gestaltung typisiert $\$ 8$ VerlG, während die Ausschließlichkeit des so erworbenen Verlagsrechtes aus $\mathbb{9}$ Abs. 2 VerlG folgt. ${ }^{102}$

Dieses aus dem Druckzeitalter überkommene Modell - umfassende Rechteübertragung der Autoren an den Verlag, verbunden mit umfassendem Rechtevorbehalt gegenüber Nutzern - dürfte in Ermangelung anderweitiger Abreden auch für Zeitschriften gelten, die zusätzlich zur gedruckten Version kostenfrei im Internet abrufbar sind. So finden sich vier rechtswissenschaftliche Internetzeitschriften (CL, FoR, HRN, ZfL), die zugleich gedruckt erscheinen und in ihrer Onlinepräsenz nicht zwischen beiden Erscheinungsweisen differenzieren. ${ }^{103}$ Dass bei diesen Zeitschriften der Absatz gedruckter Hefte durch die gleichzeitig freie Verfügbarkeit im Internet Einbußen erleiden könnte, nehmen deren Verlage wohl deshalb kommentarlos hin, weil alle vier Zeitschriften ohnehin kostendeckend aus Lehrstuhlmitteln oder durch gemeinnützige Vereine finanziert sein dürften. ${ }^{104}$ Folglich bedürften diese Zeitschriften aber auch keiner exklusiven Verwertungsrechte. Dies entbehrt insoweit nicht einer gewissen Ironie, als dazu auch die einzige rechtswissenschaftliche Internetzeitschrift gehört, die sich offen zum „linken oder alternativen Spektrum“ bekennt und die „konservative Prägung“ der traditionellen Rechtswissenschaft mit

98 Der Begriff „Nutzer“ wird hier „Lesern“ und „Abonnenten“ vorgezogen, weil Fragen der Nachnutzbarkeit nicht nur die Leser einer Veröffentlichung betreffen, sondern auch Bibliotheken und andere Nutznießer.

99 Für Bücher vgl. J. Rux, in diesem Heft, S. 70 ff.

100 Im Zuge des Open Access entstehen alternative Geschäftsmodelle, die auf einer finanziellen Kostenbeteiligung der Autoren bzw. ihrer Anstellungskörperschaften durch sog. article processing charges (APCs) beruhen.

101 Zur ökonomischen Ausgangslage konzis Ch. Engel, Give the Journals Back to the Scientists, JITE 2004, S. 35.

102 Diese „Monopolstellung soll [dem Verlag] eine möglichst gute und damit auch gewinnträchtige Verwertung ermöglichen“, so Mantz, Open Access-Lizenzen (Fn. 92), S. 96.

103 Allenfalls FoR-Redaktion: „Forum Recht ist nicht nur eine Online-Zeitschrift sondern vor allem eine ,richtige' Zeitschrift, die Abonnent_innen braucht!“ (web.archive.org/20120717005304/foru m-recht-online.de/wp).

104 Für CL der Lehrstuhl Niggli (Universität Fribourg), für die FoR der BAKJ und Forum Recht e.V., für die HRN der Hamburger Rechtsnotizen e.V., für die ZfL die Juristen-Vereinigung Lebensrecht e.V. 
provokanten Hefttiteln wie „Meins. Deins. Bürgerliche Kategorien“ (4/2017) in Frage zu stellen versucht. ${ }^{105}$

Das traditionelle Modell wird aber auch von Zeitschriften praktiziert, die eines Exklusivvertriebs zum Schutz des Druckabsatzes nicht einmal potentiell bedürfen, weil sie ausschließlich im Internet erscheinen. Sie verstehen sich zwar als kostenfreie „Internetzeitschrift“ (HFR), „Onlinepublikation“ (MIR), „Online-Journal“ (BLJ), „online erscheinende“ Zeitschrift (FreiLaw, OdW), „Online-Zeitschrift“ (DGStZ, HRRS, JSE, KriPoZ, ZIS, ZJS) oder „Zeitschrift Online“ (ZVR); ${ }^{106}$ dennoch verzichten alle Genannten auf die Open-Access-Nomenklatur. Das resultiert teils sicher aus fehlendem Problembewusstsein, ${ }^{107}$ teils wohl aber auch aus gewünschten rechtlichen Beschränkungen gegenüber Anbietern (Autoren) und Nachfragern (Nutzern). Nur eine Zeitschrift (WiJ) bezeichnet sich selbst als „Open Access" und verlangt von ihren Autoren zugleich das „ausschließliche Veröffentlichungsrecht bis zum Ablauf des Urheberrechts“. 108

Ebenso besteht ein halbes Dutzend der nicht als Open Access deklarierten Zeitschriften ganz ausdrücklich auf traditionellen Formen der Ausschließlichkeit: FreiLaw publiziert Beiträge „exklusiv“, ${ }^{109}$ der Trägerverein von OdW bedingt sich „das ausschließliche Nutzungsrecht am Manuskript aus“, 110 die „Task Force Strafrecht“ als Herausgeber von confront „das ausschließliche Verlagsrecht“ einschließlich der „Befugnis zum Speichern in einer Datenbank“ und dem „Recht der weiteren Vervielfältigung“. ${ }^{111}$ MIR und BLJ verlangen „für die Zeit bis zum Ablauf des Urheberrechts“ das „ausschließliche Nutzungs-/Verlagsrecht“ bzw. „ausschließli-

105 Vgl. Selbstverständnis FoR: „widmet es sich den Themen und Positionen, die in der gängigen juristischen oder rechtspolitischen Diskussion zu kurz kommen. Dem konservativ geprägten herrschenden Diskurs im juristischen Bereich setzen wir ein Zeitschriftenprojekt entgegen, in dem kritische Stimmen zu Wort kommen [...], die einem progressiven, linken oder alternativen Spektrum zuzurechnen sind. [...] wir sehen uns als rechtspolitische Zeitschrift, nicht als juristische Fachzeitschrift!“ (web.archive.org/20190310155818/forum-recht-online.de/wp/?page_id=111).

106 Noch vager GB („,kostenfrei und unverbindlich [...] per eMail“), GJLE („kostenfrei als PDF zum herunterladen“), Quid? („Digitale Ausgaben [...] gratis verfügbar“), StudZR-WissOn („,kostenlos im Netz abrufbar"), confront („als Newsletter versendet“), nebst ReOS und RUB RR (ohne jede Erläuterung).

107 So schon H. Hamann, Open Access in der Rechtswissenschaft, GRUR 2016, S. 1140 (1142 1. Sp.) (hanjo.1hamann.de/research/grur2016-1140.pdf); ders., Freier Zugang zur juristischen Fachliteratur im Spiegel der Open-Science-Bewegung, sui generis 3 (2016), S. 96 (103 r.Sp., Rn. 24) (sui-gene ris.ch/28).

108 Zitate von Verlagswebsite (web.archive.org/20190801140056/compliancedigital.de/short/wij/ejour nal-inhalt.html), bzw. Zeitschriftenwebsite (Nachw. wie oben Fn. 88).

109 Vgl. Impressum: web.archive.org/20120301223723/freilaw.de/impressum.

110 Vgl. Redaktion: web.archive.org/20131208055820/ordnungderwissenschaft.de/redaktion/redaktio n.htm.

111 So das Impressum zu Heft 1/2018, S. 51 in web.archive.org/20190806153509/confront.news/wp-c ontent/uploads/2018/05/confront-2018-01.pdf - gefolgt vom damit kaum vereinbaren Satz „Verlags-, Autoren- und Urheberrechte gelten nach der Lizenz der Creativ [sic] Commons [...] CC BY-NC-SA“. 
che Nutzungs- und Verwertungsrecht“, 112 und HFR immerhin das ,ausschließliche Nutzungsrecht [...] für fünf Jahre“. ${ }^{113}$ Damit setzen diese Zeitschriften also die bisher gedruckte Exklusivpublikation mit den Mitteln des Internet fort.

Soweit sich weitere 13 Internetzeitschriften weder als „Open Access“ erklären noch zum Umfang der beanspruchten Rechte äußern, ${ }^{114}$ dürften sie zwar kein exklusives Verlagsrecht erwerben $\left(\$ 31 \mathrm{~V}\right.$ UrhG) ${ }^{115}$ Wohl aber dürfte ein konkludenter Verlagsvertrag im Umfang des $\mathbb{8} 8$ VerlG i.V.m. $\mathbb{} 2$ Abs. 1 und $\$ 9$ Abs. 2 VerlG entstehen, der Nutzungsrechte allein für den Verlag begründet, ohne Dritten die Nachnutzung zu ermöglichen.

\section{III. „Open Access“ mit unklarer Rechteverteilung}

Einige Internetzeitschriften bezeichnen sich nicht nur als „Online-Zeitschrift“, sondern als „Open Access“ (vgl. Fn. 72), lassen aber entweder eine Erläuterung vermissen, welche Rechteverteilung damit beabsichtigt ist (wie HFSt, JurPC und LeGes), oder geben eine widersprüchliche Erläuterung (wie AJBALS, Cognitio und EJELS). ${ }^{116}$ Da schon die Bezeichnung „Open Access“ allerdings nach dem in der Wissenschaft etablierten Begriffsverständnis (oben bei Fn. 79) eine rechtsverbindliche Lizenzierung beinhalten könnte, bedarf diese (an Autoren wie Nutzer gleichermaßen gerichtete) Erklärung einer Auslegung nach dem objektivierten Empfängerhorizont im Sinne der $\mathbb{S} \mathbb{S} 133,157$ BGB.

Fraglich ist dann allerdings, von welchem Empfänger idealtypisch auszugehen ist: Von einem mit der herrschenden Open-Access-Definition vertrauten Empfänger, der darunter eine unwiderrufliche Lizenz zur Nachnutzung versteht? Oder von einem der Open-Access-Debatte fremden Rechtswissenschaftler, dessen Verständnis womöglich vom wissenschaftlichen Sprachgebrauch abweicht? Für letzteres spräche immerhin, dass rechtswissenschaftliche Fachzeitschriften sich primär an Autoren und Nutzer aus dem Kreis der Rechtswissenschaft richten, also für ihre Fachgemeinschaft eine eigene „restriktive Definition“ entwickelt haben könn-

112 Für MIR: web.archive.org/20140209053421/medien-internet-und-recht.de/impressum; für BLJ vgl. schon die erste Ausgabe 1/2007 (t1p.de/iwgg), S. II unter „Manuskripte“.

113 Vgl. Autorenhinweise: web.archive.org/20080619042012/humboldt-forum-recht.de/deutsch/fuer-a utoren.

114 Seltene Ausnahme: GB, deren Autorenmerkblatt (5/2010) ausdrücklich nicht ausschließliche Nutzungsrechte einfordert (web.archive.org/20190806161827/recht.uni-jena.de/z10/gb/GB-Autorenme rkblatt.pdf, unter 2.).

115 Vgl. KriPoZ-Redaktion, E-Mail v. 21.1.2019: „Autoren räumen der KriPoZ lediglich das Recht ein, den Beitrag zu veröffentlichen und über die Social Media Kanäle zu verbreiten. Alle weiteren (Nutzungs)Rechte verbleiben bei den Verfassern“.

116 Cognitio beruft sich auf die Lizenz CC BY-NC-ND im Text, auf CC BY-SA hingegen in der Grafik (web.archive.org/20190308132217/cognitio-zeitschrift.ch/impressum); AJBALS und EJELS nennen jeweils CC BY auf der Startseite, CC BY-NC-ND im Inhaltsverzeichnis, keine Lizenz dagegen in den einzelnen Beiträgen. Ebenso unklar ist die Rechtslage bei SPWR (dazu oben bei Fn. 90), die sich als „Open Access“ beschreibt, aber durch @-Vermerk gewerbliche Schutzrechte beansprucht. 
ten. ${ }^{117}$ Entscheidend dagegen spricht jedoch, dass sich eine fachspezifisch etablierte Definition gar nicht nachweisen lässt: Unter rechtswissenschaftlichen Internetzeitschriften ist eine Bandbreite von Lesarten verbreitet und die meisten Rechtswissenschaftler(innen) verbinden mit „Open Access“ wohl allenfalls vage Vorstellungen. Sie haben gerade kein konkretes, von der etablierten Definition abweichendes Begriffsverständnis. Im Gegenteil: Der im juristischen Diskurs ungewohnte Anglizismus „Open Access“ soll ja gerade den Anschluss an internationale Wissenschaftsdiskurse und ihre Publikationskultur herstellen.

Deshalb spricht mehr dafür, denjenigen als objektivierten Empfänger anzusehen, der sein Verständnis von „Open Access“ an dem in der Wissenschaft etablierten und vielerorts dokumentierten Begriffsverständnis ausrichtet. Folglich sind die oben genannten Zeitschriften im Wege der Auslegung mit echten Open-AccessZeitschriften (dazu gleich V.) gleichzustellen, um einen urheberrechtlichen Gleichlauf in beiden Konstellationen zu sichern.

\section{IV. „Open Access“ mit rechtlich restriktiver Lizenzierung}

Soweit sich rechtswissenschaftliche Internetzeitschriften als „Open Access“ erklären und zugleich eine konkrete Lizenz eindeutig benennen - oder jede Lizenz eindeutig verweigern (dazu oben bei Fn. 108) - bleibt für eine Auslegung kein Raum.

Von den eingangs (in C.I vor Tab. 2) dargestellten Lizenzen findet die DPP-Lizenz heute ${ }^{118}$ nur noch bei einer Zeitschrift Verwendung (JIPITEC). Da die von dieser Zeitschrift als Standard angebotene DPP-Basislizenz allerdings mit der Open-Access-Definition nicht vereinbar ist, ${ }^{119}$ beeinträchtigt die Wahl dieser Lizenz auch die Open-Access-Konformität der Zeitschrift insgesamt. Ein Versuch zur Gegensteuerung lässt sich vielleicht darin erkennen, dass JIPITEC es ihren Autoren freistellt, auch eine der beiden anderen DPPL-Varianten zu wählen, und sie dazu ermutigt, daneben weitere Lizenzen (bspw. CC) zu vergeben. ${ }^{120}$ Diese Gestaltung macht jedoch allenfalls einzelne Artikel Open Access verfügbar, nicht dagegen die Zeitschrift insgesamt. Damit geht die entsprechende Selbstbeschreibung fehl.

117 So Oberländer/Vock, Wissen für Alle (Fn. 67), S. 15: „In der restriktiven Definition wird Open Access als kostenfreier Zugang zu wissenschaftlichen Informationen beschrieben".

118 Bis 2015 auch JLL, vgl. Hamann, in diesem Heft, S. 150.

119 Niedersächsische SUB Göttingen (Hrsg.), Der freie Zugang zu wissenschaftlicher Information: Lizenzen, Open-Access.net: „Soweit in der Berliner Erklärung gefordert wird, dass Bearbeitungen des Lizenzgegenstandes zu ermöglichen sind, genügt die modulare DPPL jedenfalls nicht umfassend der Open Access-Definition. “ (web.archive.org/20190310212238/open-access.net/informationen-zu-op en-access/rechtsfragen/lizenzen); zum Parallelproblem bei vier der sechs CC-Lizenzen gleich noch Fn. 123.

120 JIPITEC, Applicable Open Access licenses: "The editors encourage authors to use more than one license whenever this is required by specific needs of the users not fully covered by the DPPL." (web.archive.org/20190310215807/jipitec.eu/for-authors/applicable-open-access-licenses). 
Alle übrigen Zeitschriften nutzen Standardlizenzen der CC-Initiative, wobei die Variante BY-ND nicht aufzufinden ist und zwei weitere Varianten nur von je einer Zeitschrift verwendet werden: BY-NC seit 2014 von der RiA, BY-NC-SA seit 2016 von confront. Letztere bezeichnet sich aber auch gar nicht als „Open Access“, sondern beansprucht paradoxerweise zugleich ein exklusives Verlagsrecht (vgl. Fn. 111).

Die verbreitetste Lizenz hingegen ist BY-NC-ND, die bei acht Internetzeitschriften zum Einsatz kommt (anci.ch, EJIMEL, fhi, GoJIL, Rg, VLR, ZERL und ZR\&I). Sie erfordert - wie sich aus Tab. 2 ergibt - „die Namensnennung, untersagt die kommerzielle Verwertung des lizenzierten Inhalts und die Erstellung abgeleiteter Werke“.121 Damit handelt es sich um eine „überaus restriktive“ Lizenz mit „ganz praktischen Nachteilen“, ${ }^{122}$ die ebenso wenig wie die DPP-Basislizenz als „offen“ angesehen wird, weil die etablierte Open-Access-Definition „Verbote der Bearbeitung oder kommerziellen Nutzung nicht kennt“. ${ }^{123}$ Um dennoch „sinnvoll über Open Access sprechen zu können“, schlägt die rechtswissenschaftliche Fachliteratur deshalb vor, die Lizensierung unter einer restriktiven CC-Variante ebensowenig als „Open Access“ zu bezeichnen wie „die Abrufbarkeit eines Aufsatzes über ein [...] lizenziertes Modul einer Fachdatenbank“ oder die „Veröffentlichung einer Read-Only-Version einer juristischen Monografie über Google Books“, ${ }^{124}$ die schließlich ebenfalls „mit ,Open Access“ wenig zu tun hat“. ${ }^{125}$

Allein diese Begriffsschärfung würde folglich fast die Hälfte (10 von 23) aller vermeintlich „Open Access“ publizierten rechtswissenschaftlichen Internetzeitschriften ausschließen. Zugleich erscheint sie angesichts des etablierten Sprachgebrauchs der internationalen Wissenschaftspraxis und der deutschen Wissenschaftsorganisationen unvermeidlich: Soll eine rechtssichere Auslegung möglich sein ${ }^{126}$ und die Bezeichnung „Open Access“ nicht zum beliebigen Synonym für „online“, „im Internet“ oder „irgendwie lizensiert“ degenerieren, ist die präzise Begriffsverwendung entscheidend. Nur sie beugt letztlich falschen Erwartungen der Nutzer und falschen Versprechungen der Anbieter vor.

121 Herb, Creative-Commons-Lizenzen (Fn. 96), Abs. 7.

122 L. Dobusch, Open Access bei Großverlagen: Lizenzierung zwischen liberal und restriktiv, Netzpolitik.org v. 18.12.2017 (www.netzpolitik.org/2017/open-access-b).

123 Herb, Creative-Commons-Lizenzen (Fn. 96), Abs. 10; ebenso die internationale Stiftung für freies Wissen: OKFN, Non-Conformant Licenses (web.archive.org/20190310221621/opendefinition.org/ licenses/nonconformant); zur Kritik hieran vgl. oben bei Fn. 80, zum Zweck einheitlichen Sprachgebrauchs Fn. 73.

124 Wildgans, Plädoyer (Fn. 85), S. 22 zu BY-NC-ND; im Übrigen vgl. Herb, Creative-Commons-Lizenzen (Fn. 96) und Krujatz, Open Access (Fn. 85).

125 So Bäuerle, Open Access (Fn. 85), S. 12 Fn. 57 in Reaktion auf den sog. „Heidelberger Appell“ gegen Open Access.

$126 \mathrm{Zu}$ diesem Zweck der Lizenzstandardisierung im Kontext des Open Access bspw. Fehling, Verlagsproduktion (Fn. 83), S. 344. 


\section{Open Access mit rechtlich freier Lizenzierung}

Aus den bereits dargelegten Erwägungen werden nur die beiden liberalsten CC-Lizenzen im Schrittum „als offen betrachtet“ 127 und gelten deshalb als „echte Open Access-Lizenzen ": ${ }^{128}$ Die Lizenz BY, die vom Nutzer lediglich die Urheberattribution verlangt und als „etablierter Standard für Open Access in der Wissenschaft" allgemein gilt, ${ }^{129}$ sowie die Lizenz BY-SA, die zusätzlich eine Weitergabe unter gleichen Bedingungen erfordert.

Ende 2018 nutzten nur vier rechtswissenschaftliche Internetzeitschriften eine dieser beiden Lizenzen, zum Jahresbeginn 2019 wurde auch eine der bislang restriktivsten „Open Access“-Zeitschriften (vgl. Fn. 87) entsprechend umgestellt. Damit dürfen nunmehr drei allgemeine Zeitschriften sowie eine tierschutzrechtliche und eine rechtslinguistische (dazu oben B.III) als „echte“ Open-Access-Zeitschriften gelten: ALJ, GLJ, JLL und TiRuP verwenden die CC-Lizenz mit Urheberattribution (CC BY), sui generis beschränkt zusätzlich auf die „Weitergabe unter gleichen Bedingungen" (CC BY-SA). Damit ist nun gut jede zehnte rechtswissenschaftliche Internetzeitschrift $(10,9 \%)$ zweifelsfrei Open Access. Diese Durchsetzungsrate ähnelt derjenigen anderer Geisteswissenschaften: Für Sprach- und Literaturwissenschaften wurde vor einigen Jahren eine Rate von $8,9 \%$ ermittelt, für Philosophie und Geschichtswissenschaft je $9 \%{ }^{130}$

Unter den rechtswissenschaftlichen Internetzeitschriften kommen allerdings im Wege der Auslegung noch diejenigen hinzu, die ihre Inhalte durch die Erklärung als „Open Access“ frei lizensieren, ohne jedoch den Umfang der Lizenz zweifelsfrei zu konkretisieren: AJBALS, Cognitio, EJELS, HFSt, JurPC und LeGes (dazu oben III.) dürfen nach dem maßgeblichen Empfängerhorizont ( $\mathbb{S} 133,157$ BGB) als CC-BY-lizensiert gelten, zumal diejenigen Zweifelskandidaten, die überhaupt eine Lizenzierung erwähnten, ausdrücklich auch CC BY oder CC BY-SA anführten (vgl. Fn. 116). Etwas zweifelhaft bleibt dies bei SPWR, deren Außenauftritt (dazu oben vor Fn. 90) womöglich keine Auslegung ihrer Selbstbezeichnung „Open Access“ zulässt, sondern schlicht - wie bei confront (Fn. 111) und WiJ (oben bei Fn. 108) eine paradoxe Lizenzerklärung beinhaltet.

127 Herb, Creative-Commons-Lizenzen (Fn. 96), Abs. 10; vgl. auch OKFN, Conformant Licenses (web.archive.org/20190310221621/opendefinition.org/licenses).

128 Niedersächsische SUB Göttingen (Fn. 119): „Mit Blick auf die Forderungen der Berliner Erklärung [...] handelt es sich lediglich bei den Lizenzen CC-BY und CC-BY-SA um ,echte' Open Access-Lizenzen.“; ebenso Universität Wien, Rechtsfragen zum Open-Access-Publizieren: „Von den sechs CC-Lizenzen erfüllen nur die Lizenzen CC BY und CC BY-SA die Anforderungen für ,freie Lizenzen“." (web.archive.org/20190204210830/openaccess.univie.ac.at/publikationsdienste/uscholar/rec htsfragen).

129 Dobusch, Lizenzierung (Fn. 122).

130 Herb, Creative-Commons-Lizenzen (Fn. 96), Abs. 25 aufgrund einer Auswertung des DOAJ (dazu oben bei Fn. 11); danach betrug die Quote in den Sozialwissenschaften schon $14 \%$, in der Medizin $26 \%$, in den Technikwissenschaften $29 \%$ und in den Naturwissenschaften $32 \%$. 


\section{Erfahrungsberichte und Best Practices}

Die theoretischen Überlegungen, die sich aus der Durchmusterung der rechtswissenschaftlichen Internetzeitschriften ergeben haben, sollen durch praktische Erfahrungen anschaulich kontextualisiert werden. Deshalb wurden rechtswissenschaftliche Online-Periodika eingeladen, Erfahrungsberichte beizusteuern, um Vorzüge ebenso wie Schwierigkeiten des neuen Publikationsmodells auszuloten. ${ }^{131}$ Unter den acht Onlinezeitschriften, die dieser Einladung gefolgt sind, finden sich mit ALJ, GLJ, JLL und sui generis fast alle zweifelsfreien Open-Access-Zeitschriften (oben C.V.), aber daneben auch solche, die offener Lizenzierung bisher eher fern stehen. Zugleich sind alle Rechtsgebiete vertreten: Die Spannbreite der Erfahrungsberichte reicht vom Urheberrecht (JIPITEC) bis zum Strafrecht (ZIS), von der juristischen Ausbildung (ZJS) bis zum Wissenschaftsrecht (OdW). Zudem berichtete mit dem Völkerrechtsblog auch eines der inzwischen sieben rechtswissenschaftlichen Mehrautorenblogs (dazu oben Fn. 29).

Diese Erfahrungsberichte sind so vielgestaltig ausgefallen wie die Themen der vorgestellten Zeitschriften. Eine zusammenfassende Würdigung, die über die bereits dargelegten Lizenzfragen hinausgeht, müsste wohl empfindliche Verkürzungen mit sich bringen; Verallgemeinerungen würden zwangsläufig entweder trivial oder oberflächlich ausfallen. Dies hat vereinzelt sogar Zeitschriftenherausgeber davon abgehalten, überhaupt einen Bericht beizusteuern. ${ }^{132}$ Deshalb sei den Herausgebern, die sich dennoch ins Rampenlicht gewagt haben, herzlich gedankt und nun das Wort gegeben. Aus ihren Berichten mögen sich hoffentlich Praxisstandards („Best Practices“) entwickeln, die eine kritische Rezeption von Open-Access-Texten ebenso ermöglichen wie sie etwaigen Nachahmern zur Anregung dienen können. Jedenfalls erlauben solche Erfahrungsberichte mehr Einblick in ein neues Genre, als es die unbeteiligte Analyse aus der Außenperspektive je könnte.

\section{E. Zusammenfassung und Fazit}

Als Fazit des vorliegenden Beitrags - ohne den gleich folgenden Erfahrungsberichten vorzugreifen - lässt sich festhalten, dass die Gründung juristischer Internetzeitschriften im deutschen Sprachraum in den letzten zwanzig Jahren einem stabilen, leicht positiven Trend folgte, dass diese Zeitschriften trotz hoher Varianz in Themensetzung, Erscheinungsrhythmus und Beitragssprachen bemerkenswert oft Begutachtungsverfahren (peer review) einsetzen und sich noch öfter als „Open Ac-

131 Früherer Erfahrungsbericht zur fhi bei Haferkamp u.a., Rechtsgeschichte (Fn. 45); zur GoJIL bei A. Dienelt/G. Schäfer/T. Tschappe, Völkerrecht, Peer Review und Open Access, LTO v. 20.11.2010 (www.lto.de/persistent/a_id/1984).

132 Ein Herausgeber berichtete etwa in persönlicher Korrespondenz (E-Mail v. 23.4.2019), „dass sich bei [Zeitschriftenname] vieles eher zufällig, persönlich und familiär geprägt entwickelt hat. Kurzum: Die Geschichte von [Zeitschriftenname] eignet sich nicht als ,Blaupause' für die stringente Entwicklung hin zu einem Open Access-Journal. Es mangelt an Generalisierungsfähigkeit.“. 
cess" verstehen. Dabei erfüllt allerdings kaum die Hälfte der so bezeichneten Zeitschriften tatsächlich die von den deutschen Wissenschaftsorganisationen konsentierte Definition. Wie wenig sich juristische Zeitschriftenherausgeber bislang der rechtlichen Anforderungen des Open Access bewusst sind, belegen jene drei Zeitschriften, die sich auf „Open Access“ oder eine CC-Lizenz berufen, aber zugleich alle Rechte vorbehalten („(@)) oder ein „exklusives Verlagsrecht“ bzw. „ausschließliches Veröffentlichungsrecht" beanspruchen. Betrachtet man stattdessen nur Zeitschriften als Open Access, die auch die normativen Definitionsmerkmale erfüllen, ergibt sich eine Open-Access-Quote von knapp $11 \%$ der juristischen Internetzeitschriften im deutschen Sprachraum. Das liegt ungefähr gleichauf mit anderen Geisteswissenschaften (Stand 2015) und entspricht etwa 0,6 \% (mit Zweifelsfällen 1,3\%) aller in Deutschland zitierten juristischen Fachzeitschriften (dazu Fn. 40). Die Rezeptionsneigung der Rechtswissenschaft im deutschen Raum und ihre Bereitschaft zum Anschluss an internationale Wissenschaftsdiskurse werden die weitere Entwicklung der juristischen Open-Access-Bewegung (www.jurOA.de) maßgeblich mitbestimmen. 